\title{
ON THE MOTIVIC STABLE PAIRS INVARIANTS OF $K 3$ SURFACES
}

\author{
S. KATZ, A. KLEMM, AND R. PANDHARIPANDE \\ WITH AN APPENDIX BY R. P. THOMAS
}

\begin{abstract}
For a $K 3$ surface $S$ and a class $\beta \in \operatorname{Pic}(S)$, we study motivic invariants of stable pairs moduli spaces associated to 3fold thickenings of $S$. We conjecture suitable deformation and divisibility invariances for the Betti realization. Our conjectures, together with earlier calculations of Kawai-Yoshioka, imply a full determination of the theory in terms of the Hodge numbers of the Hilbert schemes of points of $S$. The work may be viewed as the third in a sequence of formulas starting with Yau-Zaslow and KatzKlemm-Vafa (each recovering the former). Numerical data suggest the motivic invariants are linked to the Mathieu $\mathrm{M}_{24}$ moonshine phenomena.

The KKV formula and the Pairs/Noether-Lefschetz correspondence together determine the BPS counts of $K 3$-fibered Calabi-Yau 3 -folds in fiber classes in terms of modular forms. We propose a framework for a refined $\mathrm{P} / \mathrm{NL}$ correspondence for the motivic invariants of $K 3$-fibered CY 3 -folds. For the STU model, a complete conjecture is presented.
\end{abstract}

\section{Contents}

0. Introduction

1. Curve classes on $K 3$ surfaces 4

2. Stable pairs motivic invariants 5

3. Conjectures A and B 8

4. Calculation of Kawai-Yoshioka 9

5. Refined Gopakumar-Vafa invariants 11

$\begin{array}{ll}\text { 6. Conjecture C } & 15\end{array}$

$\begin{array}{ll}\text { 7. First predictions } & 18\end{array}$

8. Duality and Noether-Lefschetz theory 28

9. Summarv 38

Appendix A. Refined KKV from refined Gopakumar-Vafa 41

References

Date: September 2015. 


\section{INTRODUCTION}

A beautiful connection between curve counting on $K 3$ surfaces and modular forms was conjectured in 1995 by Yau and Zaslow [37]: the generating series of the counts of rational curves in primitive classes was conjectured to equal the inverse of the discriminant

$$
\Delta(q)=q \prod_{n=1}^{\infty}\left(1-q^{n}\right)^{24}
$$

By work of Göttsche [13], $\Delta(q)^{-1}$ was already known to arise as the generating series of the Euler characteristics of Hilbert schemes of points of $K 3$ surfaces $S$,

$$
\sum_{n \geq 0} \chi\left(\operatorname{Hilb}^{n}(S)\right) q^{n-1}=\frac{1}{\Delta(q)}
$$

An argument by Beauville [2] in 1997 provided a geometric link between curve counting in primitive classes and the Euler characteristics of $\operatorname{Hilb}^{n}(S)$.

A connection between the higher genus curve counts on $K 3$ surfaces and the generating series of $\chi_{y}$ genera of $\operatorname{Hilb}^{n}(S)$,

$$
\sum_{n \geq 0} \chi_{y}\left(\operatorname{Hilb}^{n}(S)\right) q^{n-1}=\frac{1}{q \prod_{n=1}^{\infty}\left(1-y q^{n}\right)^{2}\left(1-q^{n}\right)^{20}\left(1-y^{-1} q^{n}\right)^{2}}
$$

was proposed in 1999 [17]. The conjectures of [17] govern all classes on $K 3$ surfaces via a subtle divisibility invariance for multiple classes. In the genus 0 primitive case, the Yau-Zaslow conjecture is recovered. A proof of the KKV conjecture was recently found [30]. The moduli of sheaves (via stable pairs [27]) on $K 3$ surfaces play a central role.

We propose here a third step in the sequence of conjectures starting with Yau-Zaslow and KKV. We conjecture the Betti realization of the motivic stable pairs theory of $K 3$ surfaces is connected to the 
generating series of Hodge numbers of $\operatorname{Hilb}^{n}(S)$,

$$
\begin{gathered}
\sum_{n \geq 0} \chi_{\text {Hodge }}\left(\operatorname{Hilb}^{n}(S)\right) q^{n-1}= \\
\frac{1}{q \prod_{n=1}^{\infty}\left(1-u^{-1} y^{-1} q^{n}\right)\left(1-u^{-1} y q^{n}\right)\left(1-q^{n}\right)^{20}\left(1-u y^{-1} q^{n}\right)\left(1-u y q^{n}\right)},
\end{gathered}
$$

where the variables $u$ and $y$ keep track of the Hodge grading. Our conjecture governs all curve classes and specializes to the KKV conjecture after taking Euler characteristics.

In addition to the surprising divisibility invariance already present in the KKV conjecture [17], we propose a new deformation invariance of the Betti realization of the motivic invariants of $K 3$ geometries. To support our conjectures, we provide a few basic calculations.

The KKV formula and the Pairs/Noether-Lefschetz correspondence [25, 30] together determine the BPS counts of $K 3$-fibered Calabi-Yau 3 -folds in fiber classes in terms of modular forms. We propose a framework for a refined $\mathrm{P} / \mathrm{NL}$ correspondence for the motivic invariants of K3-fibered Calabi-Yau 3-folds. For the STU model, a complete conjecture is provided.

In the Appendix by R. Thomas, the Gopakumar-Vafa perspective on the motivic invariants of $K 3$ surfaces is discussed. The relevant moduli spaces [18] are shown to be nonsingular even in the imprimitive case. The results of the Appendix may be viewed as supporting our motivic stable pairs conjectures in the larger framework of the conjectural Pairs/Gopakumar-Vafa correspondence 1

Acknowledgements. The formulation of the conjectures (and especially of the Betti deformation invariance) was undertaken while A.K. and R.P. were visiting S.K. at the University of Illinois in April 2014.

We thank J. Bryan, J. Choi, D. Maulik, E. Scheidegger, and V. Shende for many conversations directly related to the motivic stable pairs invariants of $K 3$ surfaces. Much of the paper was written while

\footnotetext{
${ }^{1}$ An elementary overview of the various correspondences for curve counts on Calabi-Yau 3-folds can be found in [29].
} 
R.P. was attending the conference $K 3$ surfaces and their moduli on the island of Schiermonnikoog in May 2014 (organized by C. Faber, G. Farkas, and G. van der Geer with support from the Compositio foundation). Discussions there with B. Bakker, A. Bayer, D. Huybrechts, E. Macri, G. Oberdieck, and Q. Yin were very helpful. We thank G. Moore for suggesting a connection to Mathieu moonshine at String Math 2014 in Edmonton and M. Gaberdiel for related conversations.

Special thanks are due to R. Thomas for crucial help with the superpotential investigations and for contributing the Appendix on the Gopakumar-Vafa moduli approach.

S.K. was supported by NSF grant DMS-12-01089. A.K. was supported by KL 2271/1-1 and DMS-11-59265. R.P. was supported by grants SNF-200021-143274 and ERC-2012-AdG-320368-MCSK.

\section{Curve Classes on $K 3$ surfaces}

Let $S$ be a nonsingular projective $K 3$ surface. The second cohomology of $S$ is a rank 22 lattice with intersection form

$$
H^{2}(S, \mathbb{Z}) \stackrel{\cong}{=} \oplus U \oplus U \oplus E_{8}(-1) \oplus E_{8}(-1),
$$

where

$$
U=\left(\begin{array}{ll}
0 & 1 \\
1 & 0
\end{array}\right)
$$

and $E_{8}(-1)$ is the (negative) Cartan matrix. The intersection form (1.1) is even.

The divisibility $m_{\beta}$ is the maximal positive integer dividing the lattice element $\beta \in H^{2}(S, \mathbb{Z})$. If the divisibility is $1, \beta$ is primitive. Elements with equal divisibility and norm square $\langle\beta, \beta\rangle$ are equivalent up to orthogonal transformation [36].

The Picard lattice of $S$ is the intersection

$$
\operatorname{Pic}(S)=H^{2}(S, \mathbb{Z}) \cap H^{1,1}(S, \mathbb{C}) .
$$

For a family of nonsingular $K 3$ surfaces

$$
\pi: \mathcal{X} \rightarrow(\Delta, 0)
$$


with special fiber $\mathcal{X}_{0} \cong S$ and trivial local system $R^{2} \pi_{*} \mathbb{Z}$, the NoetherLefschetz locus associated to $\gamma \in H^{2}(S, \mathbb{Z})$ is

$$
N L_{\gamma}=\left\{p \in \Delta \mid \gamma \in \operatorname{Pic}\left(\mathcal{X}_{p}\right)\right\} .
$$

The Noether-Lefschetz locus is naturally a subscheme $N L_{\gamma} \subset \Delta$.

\section{Stable PAIRS MOtiviC INVARIANTS}

Let $S$ be a nonsingular projective $K 3$ surface. Curve counting on $S$ may be approached via the reduced virtual fundamental class of the moduli space of stable maps to $S$ or the stable pairs theory of CalabiYau 3-fold thickenings of $S$. An equivalence relating these two counts is proven in [30] essentially using [25, 26]. We are interested here in the motivic invariants associated to $S$. Since no motivic theory is available on the Gromov-Witten side, we will consider here the moduli spaces of stable pairs.

BPS counts for $S$ via stable pairs were defined in [30]. The construction uses $K 3$-fibrations sufficiently transverse to Noether-Lefschetz loci. We follow the geometric perspective of [30] to define a motivic theory associated to $S$.

Let $\alpha \in \operatorname{Pic}(S)$ be a nonzero class which is both positive (with respect to any ample polarization of $S$ ) and primitive. Let $T$ be a nonsingular 3-dimensional quasi-projective variety,

$$
\epsilon: T \rightarrow(\Delta, 0),
$$

fibered in $K 3$ surfaces over a pointed curve $(\Delta, 0)$ satisfying:

(i) $\Delta$ is a nonsingular quasi-projective curve with trivial canonical class,

(ii) $\epsilon$ is smooth, projective, and $T_{0} \cong S$,

The class $\alpha \in \operatorname{Pic}(S)$ is $m$-rigid with respect to the family $\epsilon$ if the following further condition is satisfied: 
(*) for every effective decomposition 2

$$
m \alpha=\sum_{i=1}^{l} \gamma_{i} \in \operatorname{Pic}(S),
$$

the local Noether-Lefschetz locus $\mathrm{NL}\left(\gamma_{i}\right) \subset \Delta$ corresponding to each class $\gamma_{i} \in \operatorname{Pic}(S)$ is the reduced point $0 \in \Delta$.

Let $\operatorname{Eff}(m \alpha) \subset \operatorname{Pic}(S)$ denote the subset of effective summands of $m \alpha$. The existence of $m$-rigid families is easy to see [30, Section 6.2].

Assume $\alpha$ is $m$-rigid with respect to the family $\epsilon$. By property $(\star)$, there is a compact, open, and closed component

$$
P_{n}^{\star}(T, \gamma) \subset P_{n}(T, \gamma)
$$

parameterizing stable pairs 3 supported set-theoretically over the point $0 \in \Delta$ for every effective summand $\gamma \in \operatorname{Eff}(m \alpha)$. We define

$$
\mathrm{W}_{n, \gamma}^{\star}(T) \in \mathrm{K}_{\mathrm{var}}^{\widehat{\mu}}\left[\mathrm{L}^{-1}\right]
$$

to be the motivid 4 stable pairs invariant associated to the component $P_{n}^{\star}(T, \gamma)$ following Joyce and collaborators [4].

The motivic invariant (2.1) takes values in the Grothendieck ring of varieties carrying actions of groups of $n^{\text {th }}$ roots of unity $\mathrm{K}_{\mathrm{var}}^{\widehat{\mu}}$ extended by the inverse of the Tate class,

$$
L=\left[\mathbb{A}^{1}\right] .
$$

The product in $\mathrm{K}_{\mathrm{var}}^{\widehat{\mu}}$ is not induced by the ordinary product of varieties, but rather defined explicitly by motivic convolution with a Fermat curve [9, 24].

\footnotetext{
${ }^{2}$ An effective decomposition requires all parts $\gamma_{i}$ to be effective divisors.

${ }^{3}$ For any class $\gamma \in \operatorname{Pic}(S)$, we denote the push-forward to $H_{2}(T, \mathbb{Z})$ also by $\gamma$. Let $P_{n}(T, \gamma)$ be the moduli space of stable pairs of Euler characteristic $n$ and class $\gamma \in H_{2}(T, \mathbb{Z})$.

${ }^{4}$ At the moment, $\mathrm{W}_{n, \gamma}^{\star}(T)$ is defined only after a choice of orientation data is made. Our discussion implicitly assumes either that there is a canonical choice or that the choice does not affect the motivic class for our $K 3$ geometry (or, at the very least, does not affect the Poincaré polynomials of the motivic classes here). Certainly $P_{n}^{\star}(T, \gamma)$ is often simply connected. If $\pi_{1}$ is trivial, then the orientation is unique. Perhaps $P_{n}^{\star}(T, \gamma)$ is always simply connected?
} 
Let $\rho$ denote the canonical action of the finite group scheme

$$
\mu_{2}=\{ \pm 1\}
$$

on itself. We obtain an element

$$
\left[\mu_{2}, \rho\right] \in \mathrm{K}_{\mathrm{var}}^{\widehat{\mu}} .
$$

Straightforward calculation using the definition of the product or the motivic Thom-Sebastiani formula yields the relation

$$
\left(1-\left[\mu_{2}, \rho\right]\right)^{2}=\mathrm{L},
$$

so we define $L^{\frac{1}{2}}$ by

$$
\mathrm{L}^{\frac{1}{2}}=1-\left[\mu_{2}, \rho\right],
$$

see [23, Remark 19]. The ring $\mathrm{K}_{\text {var }}^{\widehat{\mu}}\left[\mathrm{L}^{-1}\right]$ therefore contains all powers of $\mathrm{L}^{ \pm 1 / 2}$.

Definition. Let $\alpha \in \operatorname{Pic}(S)$ be a primitive, positive class. Given a family $\epsilon: T \rightarrow(\Delta, 0)$ satisfying conditions (i), (ii), and ( $\star$ ) for $m \alpha$, let

$$
\begin{aligned}
\sum_{n \in \mathbb{Z}} \mathbf{V}_{n, m \alpha}^{\epsilon}(S) q^{n} & = \\
\operatorname{Coeff}_{v^{m \alpha}} & {\left[\log \left(1+\sum_{n \in \mathbb{Z}} \sum_{\gamma \in \operatorname{Eff}(m \alpha)} q^{n} v^{\gamma} \mathbf{W}_{n, \gamma}^{\star}(T)\right)\right] . }
\end{aligned}
$$

The motivic invariant $\bigvee_{n, m \alpha}^{\epsilon}(S)$ is the main topic of the paper. The superscript $\epsilon$ records the family

$$
\epsilon: T \rightarrow(\Delta, 0)
$$

used in the definition.

For positive $\beta \in \operatorname{Pic}(S)$, we may write $\beta=m \alpha$ where $\alpha \in \operatorname{Pic}(S)$ is positive and primitive and $m=m_{\beta}$ is the divisibility of $\beta$. Hence,

$$
\mathrm{V}_{n, \beta}^{\epsilon}(S)=\mathrm{V}_{n, m \alpha}^{\epsilon}(S)
$$

is defined. 


\section{Conjectures A And B}

3.1. Poincaré polynomial. We formulate here several conjectures and speculations concerning the motivic stable pairs invariants $V_{n, \beta}^{\epsilon}(S)$ introduced in Section 2, Let

$$
\mathrm{H}_{n, \beta}^{\epsilon}(S) \in \mathbb{Q}[u]
$$

denote the virtual Poincaré polynomial of the motivic invariant $\bigvee_{n, \beta}^{\epsilon}(S)$.

Conjecture A. The virtual Poincaré polynomial $\mathrm{H}_{n, \beta}^{\epsilon}(S)$ is independent of the family

$$
\epsilon: T \rightarrow(\Delta, 0)
$$

satisfying conditions (i), (ii), and ( $\star$ ) for $\alpha=\frac{1}{m_{\beta}} \beta$ and $m=m_{\beta}$.

Assuming the validity of Conjecture A, we may drop the $\epsilon$ superscript and write $\mathrm{H}_{n, \beta}(S)$ for the virtual Poincaré polynomial.

Conjecture B. The virtual Poincaré polynomial $\mathrm{H}_{n, \beta}(S)$ is invariant under deformations of $S$ for which $\beta$ remains algebraic.

The divisibility $m_{\beta}$ and the norm square

$$
\langle\beta, \beta\rangle=2 h-2
$$

are the only deformation invariants of the pair $(S, \beta)$ with $\beta \in \operatorname{Pic}(S)$. Assuming the validity of Conjecture $\mathrm{B}$, we write

$$
\mathrm{H}_{n, \beta}(S)=\mathrm{H}_{n, m_{\beta}, h},
$$

replacing $(S, \beta)$ by $m_{\beta}$ and $h$.

3.2. Motives. Conjecture A for the motivic invariant $\mathrm{V}_{n, \beta}^{\epsilon}(S)$ seems not unreasonable: $\mathrm{V}_{n, \beta}^{\epsilon}(S)$ is independent of $\epsilon$. However, Conjecture $\mathrm{B}$ is certainly false with $\bigvee_{n, \beta}^{\epsilon}(S)$ in place of $\mathrm{H}_{n, \beta}(S)$ since the class

$$
[S] \in \mathrm{K}_{\mathrm{var}}
$$

often appears in $\mathrm{V}_{n, \beta}^{\epsilon}(S)$. Examples of the latter phenomenon can be found already for elliptically fibered $K 3$ surfaces in the fiber class. 
The classes of $K 3$ surfaces derived equivalent to $S$ also appear in the elliptically fibered cases.

We define a new quotient ring of varieties:

$$
\mathrm{K}_{\mathrm{var}}^{D}=\mathrm{K}_{\mathrm{var}}^{\widehat{\mu}} / \mathrm{I}^{D},
$$

where $\mathrm{I}^{D}$ is the ideal generated by all differences

$$
[X]-[\widetilde{X}] \in \mathrm{K}_{\text {var }}^{\widehat{\mu}}
$$

where $X$ and $\tilde{X}$ are $K 3$ surfaces which are derived equivalent.5 Then, we could hope the class

$$
\overline{\mathrm{V}}_{n, m \alpha}^{\epsilon}(S) \in \mathrm{K}_{\text {var }}^{D}\left[\mathrm{~L}^{-1}\right],
$$

obtained from $\mathrm{V}_{n, m \alpha}^{\epsilon}(S)$, is a universal polynomial in the motivic powers of the class of the underlying $K 3$ surface,

$$
\overline{\left[\mathrm{Sym}^{r} S\right]} \in \mathrm{K}_{\text {var }}^{D}\left[\mathrm{~L}^{-1}\right] .
$$

The coefficients of such a polynomial would lie in $\mathbb{Q}\left[L, L^{-1}\right]$.

An interesting related question immediately arises. Let $S$ be nonsingular projective $K 3$ surface with a positive irreducible class $\alpha \in \operatorname{Pic}(S)$. The moduli space $P_{n}(S, \alpha)$ is known to be nonsingular [20, 28]. Is there a formula for

$$
\overline{\left[P_{n}(S, \alpha)\right]} \in \mathrm{K}_{\text {var }}^{D}\left[\mathbf{L}^{-1}\right]
$$

as a universal polynomial in the motivic powers $\overline{\left[\mathrm{Sym}^{r} S\right]}$ ?

While we have (modest) computational evidence for Conjectures A and B constraining $\mathrm{H}_{m, \beta}^{\epsilon}(S)$, the above hopes for $\mathrm{V}_{m, \beta}^{\epsilon}(S)$ are simply speculations.

\section{Calculation of Kawai-Yoshioka}

In order to formulate Conjecture $\mathrm{C}$ which completely determines $\mathrm{H}_{n, m, h}$, we first review the calculation of Kawai-Yoshioka.

\footnotetext{
${ }^{5}$ Or perhaps $\mathrm{I}^{D}$ should be the ideal generated by the differences of all derived equivalent nonsingular projective Calabi-Yau varieties. Alternatively, Chow motives may be a more natural framework for the entire discussion.
} 
Let $P_{n}(S, h)$ denote the moduli space of stable pairs on the $K 3$ surface $S$ for a positive irreducible class $\alpha$ satisfying

$$
2 h-2=\langle\alpha, \alpha\rangle .
$$

The moduli space $P_{n}(S, h)$ is nonsingular of dimension $2 h-1+n$. The Poincaré polynomial6 of $P_{n}(S, h)$,

$$
\widetilde{\mathrm{H}}\left(P_{n}(S, h)\right)=\sum_{i=0}^{2 h-1+n} \operatorname{dim}_{\mathbb{Q}} H^{2 i}\left(P_{n}(S, h), \mathbb{Q}\right) u^{2 i} \in \mathbb{Z}[u],
$$

has been calculated by Kawai-Yoshioka. By Theorem 5.158 of [20],

$$
\begin{aligned}
& \left(u^{2} \tilde{y}-1\right)\left(1-\tilde{y}^{-1}\right) \cdot \sum_{h=0}^{\infty} \sum_{n=1-h}^{\infty} \widetilde{\mathrm{H}}\left(P_{n}(S, h)\right) u^{-2 h} \tilde{y}^{n} q^{h}= \\
& \quad \prod_{n=1}^{\infty} \frac{1}{\left(1-\tilde{y} q^{n}\right)\left(1-u^{2} \tilde{y} q^{n}\right)\left(1-q^{n}\right)^{20}\left(1-\tilde{y}^{-1} q^{n}\right)\left(1-\left(u^{2} \tilde{y}\right)^{-1} q^{n}\right)} .
\end{aligned}
$$

In order to fit our motivic conventions in Section 6, we define

$$
\mathrm{H}\left(P_{n}(S, h)\right)=u^{-2 h+1-n} \widetilde{\mathbf{H}}\left(P_{n}(S, h)\right)
$$

and rewrite the Kawai-Yoshioka formula as

$$
\begin{aligned}
& u^{-1}\left(u^{2} \tilde{y}-1\right)\left(1-\tilde{y}^{-1}\right) \cdot \sum_{h=0}^{\infty} \sum_{n=1-h}^{\infty} \mathrm{H}\left(P_{n}(S, h)\right) u^{n} \tilde{y}^{n} q^{h}= \\
& \quad \prod_{n=1}^{\infty} \frac{1}{\left(1-\tilde{y} q^{n}\right)\left(1-u^{2} \tilde{y} q^{n}\right)\left(1-q^{n}\right)^{20}\left(1-\tilde{y}^{-1} q^{n}\right)\left(1-\left(u^{2} \tilde{y}\right)^{-1} q^{n}\right)} .
\end{aligned}
$$

After the substitution $y=u \tilde{y}$, we find

$$
\begin{aligned}
& (u y-1)\left(u^{-1}-y^{-1}\right) \cdot \sum_{h=0}^{\infty} \sum_{n=1-h}^{\infty} \mathrm{H}\left(P_{n}(S, h)\right) y^{n} q^{h}= \\
& \prod_{n=1}^{\infty} \frac{1}{\left(1-u^{-1} y^{-1} q^{n}\right)\left(1-u^{-1} y q^{n}\right)\left(1-q^{n}\right)^{20}\left(1-u y^{-1} q^{n}\right)\left(1-u y q^{n}\right)} .
\end{aligned}
$$

The right side of the above formula is the generating series of Hodge polynomials of the Hilbert schemes of points of $S$.

\footnotetext{
${ }^{6}$ All the cohomology of $P_{n}(S, h)$ is even.
} 


\section{ReFined Gopakumar-VAFA InVARIAnts}

5.1. Definition. Refined Gopakumar-Vafa invariants were defined mathematically in [7] via refined stable pairs invariants.7 Following [7], we will define refined invariants

$$
\mathrm{R}_{j_{L}, j_{R}}^{h} \in \mathbb{Z}
$$

for $K 3$ surfaces for all $h \in \mathbb{Z}$ and all half-integers

$$
j_{L}, j_{R} \in \frac{1}{2} \mathbb{Z}_{\geq 0}=\left\{0, \frac{1}{2}, 1, \frac{3}{2}, \ldots\right\} .
$$

The definition uses formula (8.1) of [7] and the generating series of Hodge numbers of the Hilbert schemes of points of $K 3$ sufaces of Section 4. If $h<0$, the definition is simple:

$$
\mathrm{R}_{j_{L}, j_{R}}^{h<0}=0 .
$$

For the $h \geq 0$ case, we will use the following notation. For $j \in \frac{1}{2} \mathbb{Z}_{\geq 0}$, we define

$$
[j]_{x}=x^{-2 j}+x^{-2 j+2}+\cdots+x^{2 j-2}+x^{2 j} .
$$

We define the refined invariants $\mathrm{R}_{j_{L}, j_{R}}^{h \geq 0}$ for $K 3$ surfaces by

$$
\begin{aligned}
& \sum_{h=0}^{\infty} \sum_{j_{L}} \sum_{j_{R}} \mathrm{R}_{j_{L}, j_{R}}^{h}\left[j_{L}\right]_{y}\left[j_{R}\right]_{u} q^{h}= \\
& \prod_{n=1}^{\infty} \frac{1}{\left(1-u^{-1} y^{-1} q^{n}\right)\left(1-u^{-1} y q^{n}\right)\left(1-q^{n}\right)^{20}\left(1-u y^{-1} q^{n}\right)\left(1-u y q^{n}\right)} .
\end{aligned}
$$

Here, the sums over $j_{L}$ and $j_{R}$ are both taken over $\frac{1}{2} \mathbb{Z}_{\geq 0}$.

The above definition of $\mathrm{R}_{j_{L}, j_{R}}^{h}$ will be discussed further in Section 6. Formula (5.1) will be shown there to be consistent with the KawaiYoshioka calculation of Section 4 via the definition of the refined invariants in [7]. In fact, consistency with the Kawai-Yoshioka calculation forces formula (5.1) for $\mathrm{R}_{j_{L}, j_{R}}^{h}$.

\footnotetext{
${ }^{7}$ Other definition has been proposed in [14,21]. Connecting the refined invariants for $K 3$ surfaces $\mathrm{R}_{j_{L}, j_{R}}^{h}$ defined here to the geometry of [14, 21] is the topic of the Appendix by R. Thomas.
} 
The refined invariants $\mathrm{R}_{j_{L}, j_{R}}^{h}$ may be viewed as arising from the cohomology of $P_{n}(S, h)$, the moduli space of stable pairs on the $K 3$ surface $S$ with positive irreducible class $\alpha$ satisfying

$$
\langle\alpha, \alpha\rangle=2 h-2 \text {. }
$$

Formula (5.1) also agrees with the refined invariant for $K 3$ surfaces calculated in [15].8

Formula (5.1) uniquely determines $\mathrm{R}_{j_{L}, j_{R}}$ for $h \geq 0$. As a consequence, the following stabilization property holds: for fixed $i$ and $j$, the refined invariant $\mathrm{R}_{\frac{h}{2}-i, \frac{h}{2}-j}^{h}$ is independent of $h$ for sufficiently large $h .9$

\begin{tabular}{|c|c|}
\hline $\mathrm{R}_{\frac{i}{2}, \frac{j}{2}}^{0}$ & $\mathrm{i}=0$ \\
\hline $\mathrm{j}=0$ & 1 \\
\hline
\end{tabular}

\begin{tabular}{|c|cc|}
\hline $\mathrm{R}_{\frac{i}{2}, \frac{j}{2}}^{1}$ & $\mathrm{j}=0$ & 1 \\
\hline $\mathrm{i}=0$ & 20 & \\
1 & & 1 \\
\hline
\end{tabular}

\begin{tabular}{|c|ccc|}
\hline $\mathrm{R}_{\frac{i}{2}, \frac{j}{2}}^{2}$ & $\mathrm{j}=0$ & 1 & 2 \\
\hline $\mathrm{i}=0$ & 231 & & \\
1 & & 21 & \\
2 & & & 1 \\
\hline
\end{tabular}

\begin{tabular}{|c|cccc|}
\hline $\mathrm{R}_{\frac{i}{2}, \frac{j}{2}}^{3}$ & $\mathrm{j}=0$ & 1 & 2 & 3 \\
\hline $\mathrm{i}=0$ & 1981 & & 1 & \\
1 & & 252 & & \\
2 & 1 & & 21 & \\
3 & & & & 1 \\
\hline
\end{tabular}

\begin{tabular}{|c|ccccc|}
\hline $\mathrm{R}_{\frac{i}{2}, \frac{j}{2}}^{4}$ & $\mathrm{j}=0$ & 1 & 2 & 3 & 4 \\
\hline $\mathrm{i}=0$ & 13938 & & 21 & & \\
1 & & 2233 & & 1 & \\
2 & 21 & & 253 & & \\
3 & & 1 & & 21 & \\
4 & & & & & 1 \\
\hline
\end{tabular}

TABLE 1. All nonvanishing $\mathrm{R}_{j_{L}, j_{R}}^{h}$ for $h \leq 4$ for $K 3$ surfaces

5.2. Unrefined BPS invariants. Let $X$ be a nonsingular projective Calabi-Yau 3-fold, and let $\beta \in H_{2}(X, \mathbb{Z})$ be a curve class.

The relation of the refined BPS invariants $\mathrm{N}_{j_{L} j_{R}}^{\beta}$ of $X$ to the unrefined BPS invariants $\mathrm{n}_{g}^{\beta}$ of $X$ is obtained by from their definitions in terms

\footnotetext{
${ }^{8}$ See equations (8.3) and (8.4) of $[15]$ as well as Table 8 in Appendix D.2.

${ }^{9}$ Refined invariants for local $\mathbb{P}^{2}$ were found to stabilize in [CKK]. We expect stabilization to hold more generally.
} 
of traces on the BPS Hilbert space $\mathcal{H}_{B P S}$ arising from wrapping $M 5$ branes on curves in $X$. The Hilbert space $\mathcal{H}_{B P S}$ carries an

$$
S U(2) \times S U(2)=S U(2)_{L} \times S U(2)_{R}
$$

action. We denote the irreducible representations of $S U(2) \times S U(2)$ by pairs $\left[j_{L}, j_{R}\right]$ where $j_{L}, j_{R} \in \frac{1}{2} \mathbb{Z}_{\geq 0}$. The refined invariants arise via the formula:

$$
\operatorname{Tr}_{\mathcal{H}_{B P S}} y^{\sigma_{3}^{L}} u^{\sigma_{3}^{R}} q^{H}=\sum_{\beta} \sum_{j_{L}, j_{R} \in \frac{1}{2} \mathbb{Z}_{\geq 0}} \mathrm{~N}_{j_{L}, j_{R}}^{\beta}\left[j_{L}\right]_{y}\left[j_{R}\right]_{u} q^{\beta} .
$$

Similarly, the unrefined invariants arise as:

$$
\operatorname{Tr}_{\mathcal{H}_{B P S}}(-1)^{F_{R}} y^{\sigma_{3}^{L}} q^{H}=\sum_{\beta} \sum_{g \in \mathbb{Z}_{\geq 0}} \mathrm{n}_{g}^{\beta}\left(y^{\frac{1}{2}}+y^{-\frac{1}{2}}\right)^{2 g} q^{\beta} .
$$

Here, $(-1)^{F_{R}}$ acts as $(-1)^{2 j_{R}}$ on $\left[j_{R}\right]$. The matrix

$$
\sigma_{3}=\left(\begin{array}{cc}
1 & 0 \\
0 & -1
\end{array}\right)
$$

is twice the Cartan element of $S U(2)$ measuring the spins of the BPS state. The operator $H$ measures the mass of the BPS state.

Let $I_{g}$ denote the $S U(2)$ representation associated to the cohomology of an abelian variety of dimension $g$,

$$
I_{g}=\left(2[0]+\left[\frac{1}{2}\right]\right)^{\otimes g} .
$$

By taking the trace on $I_{g}$, we obtain the following identity:

$$
\operatorname{Tr}_{I_{g}} y^{\sigma_{3}}=\left(y^{\frac{1}{2}}+y^{-\frac{1}{2}}\right)^{2 g} .
$$

Setting $u=-1$ in (5.2) and using (5.3), we obtain the basic relationship between the refined and unrefined invariants:

$$
\sum_{j_{L}, j_{R} \in \frac{1}{2} \mathbb{Z}_{\geq 0}}(-1)^{2 j_{R}}\left(2 j_{R}+1\right) \mathrm{N}_{j_{L}, j_{R}}^{\beta}\left[j_{L}\right]=\sum_{g \in \mathbb{Z}_{\geq 0}} \mathrm{n}_{g}^{\beta} I_{g} .
$$

If we specialize (5.4) to the refined invariants $\mathrm{R}_{j_{L}, j_{R}}^{h}$ of $K 3$ surfaces and change variables

$$
y \mapsto-y
$$


in (5.1), we obtain

$$
\sum_{j_{L}, j_{R} \in \frac{1}{2} \mathbb{Z}_{\geq 0}}\left(2 j_{R}+1\right) \mathrm{R}_{j_{L}, j_{R}}^{h}\left[j_{L}\right]_{y}=\sum_{g \in \mathbb{Z}_{\geq 0}}(-1)^{g} \mathrm{r}_{g}^{h}\left(y^{\frac{1}{2}}-y^{-\frac{1}{2}}\right)^{2 g}
$$

and recover the KKV formula for the BPS counts $\mathrm{r}_{g}^{h}$ of $K 3$ surfaces from (5.1),

$$
\begin{aligned}
\sum_{h=0}^{\infty} \sum_{g \geq 0}(-1)^{g} \mathrm{r}_{g}^{h}\left(y^{\frac{1}{2}}-y^{-\frac{1}{2}}\right)^{2 g} q^{h}= \\
\\
\quad \prod_{n=1}^{\infty} \frac{1}{\left(1-y^{-1} q^{n}\right)\left(1-y q^{n}\right)\left(1-q^{n}\right)^{20}\left(1-y^{-1} q^{n}\right)\left(1-y q^{n}\right)},
\end{aligned}
$$

in accordance with [25, 30].

\begin{tabular}{|c|ccccc|}
\hline $\mathrm{r}_{g}^{h}$ & $h=0$ & 1 & 2 & 3 & 4 \\
\hline$g=0$ & 1 & 24 & 324 & 3200 & 25650 \\
1 & & -2 & -54 & -800 & -8550 \\
2 & & & 3 & 88 & 1401 \\
3 & & & & -4 & -126 \\
4 & & & & & 5 \\
\hline
\end{tabular}

TABLE 2. All nonvanishing $\mathrm{r}_{g}^{h}$ for $h \leq 4$ for $K 3$ surfaces

5.3. Mathieu moonshine. The important conceptual difference between the invariants $\mathrm{N}_{j_{L}, j_{R}}^{\beta}$ and $\mathrm{n}_{g}^{\beta}$ is that the former are actual BPS degeneracies. Hence, we expect $\mathrm{N}_{j_{L}, j_{R}}^{\beta}$ to always be nonnegative. Moreover, if there is a symmetry acting on the Hilbert space which commutes with $H$ and the spin operators, the multiplicities must fall in representations of the symmetry group. For $E_{n}$ del Pezzo surfaces, the invariants $\mathbf{N}_{j_{r} j_{R}}^{\beta}$ were observed in [15] to fall naturally in representations of $E_{n}$. The Weyl group of $E_{n}$ acts on the geometry by Lefshetz monodromy.

Greg Moore pointed out to us at String Math 2014 during the presentation [19] that the number $\mathrm{R}_{0,0}^{2}=231$ is the dimension of an irreducible representation of the Matthieu group $\mathrm{M}_{24}$, a sporadic group of order 
244823040, which is conjecturally [11] related to the elliptic genus of the $K 3$ surface. The dimensions of the 26 irreducible representations of the group $\mathrm{M}_{24}$ are

$$
\begin{aligned}
& 1,23,45,231,252,253,483,770,990,1035,1265, \\
& 1771,2024,2277,3312,3520,5313,5544,5796,10395,
\end{aligned}
$$

where the representations of dimension 45, 231,770,990, 1035 come in complex conjugated pairs. There is an extra real representation of dimension 1035.

We have checked all the values taken by refined invariants $\mathrm{R}_{j_{L}, j_{R}}^{h}$ in Table 1, except for the values 20 (and $21=20+1$ ), are expressible in a simple way in terms of the dimensions of the irreducible representations of $\mathrm{M}_{24}$. Beside the dimensions which occur directly, we have:

$$
\begin{array}{lll}
\mathrm{R}_{0,0}^{3} & =1981=2 \cdot 990+1 & \mathrm{R}_{0,0}^{4}=13938=2 \cdot 5313+3312 \\
\mathrm{R}_{\frac{1}{2}, \frac{1}{2}}^{4}=2233=2 \cdot 990+253 & \mathrm{R}_{1,1}^{5}=2254=1771+483 \\
\mathrm{R}_{\frac{3}{2}, \frac{3}{2}}^{6}=2255=1265+990 . & &
\end{array}
$$

Since the dimensions of the representations of $\mathrm{M}_{24}$ are small, the significance is somewhat limited. The decompositions with a minimal numbers of summands given above are not always unique. For example,

$$
\mathrm{R}_{\frac{3}{2}, \frac{3}{2}}^{6}=2024+231 \text { and } \mathrm{R}_{0,0}^{4}=10395+3520+23 .
$$

Nevertheless, the action of the Matthieu group is expected 10 in the string compactification on $K 3 \times T^{2}$ and the refined spacetime BPS spectrum is a natural place to see its action.

\section{Conjecture C}

Our motivic convention is the following 11 If the moduli space of stable pairs $P$ is a nonsingular variety of complex dimension $d$, then

\footnotetext{
${ }^{10}$ See [5, [12 for related constructions and [6] for subsequent developments.

${ }^{11}$ Our conventions here distribute the signs in a slightly different manner than the conventions of $[7$, but there is no essential difference.
} 
the associated motivic invariant is defined 12 to be

$$
\mathrm{L}^{-\frac{d}{2}}[P] \in \mathrm{K}_{\text {var }}^{\widehat{\mu}}\left[\mathrm{L}^{-1}\right] .
$$

Here, $[P]$ is the usual element associated to $P$ in the Grothendieck ring of varieties. When considering the virtual Poincaré polynomial,

$$
\widetilde{\mathrm{H}}: \mathrm{K}_{\text {var }}^{\widehat{\mu}}\left(\mathrm{L}^{-1}\right) \rightarrow \mathbb{Z}\left[u, u^{-1}\right],
$$

we map $\mathrm{L}^{ \pm \frac{1}{2}} \mapsto u^{ \pm 1}$. We define $\mathrm{H}(P)$ to be the virtual Poincaré polynomial of $\mathrm{L}^{-\frac{d}{2}}[P]$.

Let $\alpha \in \operatorname{Pic}(S)$ be a positive irreducible class of norm square

$$
\langle\alpha, \alpha\rangle=2 h-2 .
$$

Then $P_{n}(S, h)$ is nonsingular of dimension $2 h-1+n$. Hence, the relation

$$
\mathbf{H}\left(P_{n}(S, h)\right)=u^{-2 h+1-n} \widetilde{\mathbf{H}}\left(P_{n}(S, h)\right)
$$

of Section 4 is consistent with our motivic conventions.

An elementary verification based upon the interpretation of the KawaiYoshioka calculation in Section 4 and the definition of the refined invariants in Section 5 yields the following identity: the $v^{\alpha}$ coefficient of the product

$$
\prod_{j_{L}, j_{R}, m_{L}, m_{R}, m, j}\left(1+u^{-m+1+2 j-2 m_{R}} y^{m-2 m_{L}} v^{\alpha}\right)^{(-1)^{2\left(j_{L}+j_{R}\right)} \mathrm{R}_{j_{L}, j_{R}}^{h}}
$$

exactly equals

$$
\sum_{n=1-h}^{\infty} \mathrm{H}\left(P_{n}(S, h)\right) y^{n}
$$

The product $\prod_{j_{L}, j_{R}, m_{L}, m_{R}, m, j}$ in (6.1) signifies

$$
\prod_{j_{L} \in \frac{1}{2} \mathbb{Z}_{\geq 0}} \prod_{j_{L} \in \frac{1}{2} \mathbb{Z}_{\geq 0}} \prod_{m_{L}=-j_{L}}^{j_{L}} \prod_{m_{R}=-j_{R}}^{j_{R}} \prod_{m=1}^{\infty} \prod_{j=0}^{m-1}
$$

where $m_{L}$ and $m_{R}$ increase by steps of 1 .

\footnotetext{
${ }^{12}$ The motivic invariant of $P$ may depend on a choice of orientation. We have made an implicit choice here as explained in Section (7.1) - the only choice possible if $\operatorname{Pic}(P)$ has no 2-torsion.
} 
The product (6.1) occurs in the definition of the refined invariants [7. Equation (8.1)]. The equality of (6.1) and (6.2) is a geometric constraint verified by definition (5.1). In fact, definition (5.1) is uniquely determined by the above constraint.

To state our last conjecture, let $\alpha \in \operatorname{Pic}(S)$ be a positive, primitive class of norm square

$$
\langle\alpha, \alpha\rangle=2 h-2 .
$$

We will consider the motivic partition function for classes which are multiples of $\alpha$,

$$
\mathrm{Z}_{h}=\exp \left(\sum_{k=1}^{\infty} \sum_{n \in \mathbb{Z}} \mathrm{H}_{n, k \alpha} y^{n} v^{k \alpha}\right) .
$$

For fixed $k$, the motivic invariant $\mathrm{H}_{n, k \alpha}$ vanishes for sufficiently negative $n$. Assuming Conjectures A and B, we rewrite the partition function as

$$
\mathbf{Z}_{h}=\exp \left(\sum_{k=1}^{\infty} \sum_{n \in \mathbb{Z}} \mathbf{H}_{n, k, h[k]} y^{n} v^{k}\right)
$$

where we define

$$
2 h[k]-2=\langle k \alpha, k \alpha\rangle=k^{2}(2 h-2), \quad h[k]=k^{2}(h-1)+1 .
$$

The variable $v^{\alpha}$ has now been replaced by just $v$.

Conjecture C. For all $h$, the partition function

$$
\mathrm{Z}_{h}=\exp \left(\sum_{k=1}^{\infty} \sum_{n \in \mathbb{Z}} \mathrm{H}_{n, k, h[k]} y^{n} v^{k}\right)
$$

equals the product

$$
\prod_{k=1}^{\infty} \prod_{j_{L}, j_{R}, m_{L}, m_{R}, m, j}\left(1+u^{-m+1+2 j-2 m_{R}} y^{m-2 m_{L}} v^{k}\right)^{(-1)^{2\left(j_{L}+j_{R}\right)} \mathrm{R}_{j_{L}, j_{R}}^{h[k]}} .
$$

The product $\prod_{j_{L}, j_{R}, m_{L}, m_{R}, m, j}$ appearing in Conjecture $\mathrm{C}$ is just as before (6.3). Conjecture $\mathrm{C}$ determines every $\mathrm{H}_{n, k, h[k]}$ in terms of the 
refined Gopakumar-Vafa invariants obtained from primitive class geometry. Such a relation may be viewed as a divisibility invariance property.

If we substitute $u=-1$ in $\mathrm{H}_{n, k \alpha}$, we recover the stable pairs invariants $R_{n, k \alpha}$ defined in [30] for $K 3$ surfaces. An unwinding of the definitions then shows Conjecture $\mathrm{C}$ implies the KKV conjecture (proven in [30]) for stable pairs invariants in all classes.

\section{First PREDictions}

7.1. Virtual motives. Before presenting examples, we quickly review the theory of virtual motives following [4, 16].

Joyce and collaborators introduce the notion of an oriented d-critical locus as a framework for defining motivic invariants within classical (non-derived) algebraic geometry. Moduli spaces of stable pairs carry such a structure. We review the aspects which are most relevant for us and refer the reader to [16] for the omitted details.

A $d$-critical locus is a variety $M$ which can locally be realized as Crit $(f)$ for a holomorphic $f$ on a smooth space $U$ (a critical chart), with a weak notion of compatibility among the critical charts. The compatibility is strong enough however to define a virtual canonical bundle

$$
K_{M}^{\mathrm{vir}} \in \operatorname{Pic}\left(M^{\mathrm{red}}\right) .
$$

Given a critical chart $(U, f)$, there is a canonical isomorphism

$$
\left.\left.K_{M}^{\mathrm{vir}}\right|_{\operatorname{Crit}(f)} \simeq K_{U}^{\otimes 2}\right|_{\operatorname{Crit}(f)} .
$$

An orientation is a choice of square root of the virtual canonical bundle

$$
\left(K_{M}^{\mathrm{vir}}\right)^{1 / 2} \in \operatorname{Pic}\left(M^{\mathrm{red}}\right) .
$$

From the data of a oriented $d$-critical locus, a virtual motive $[M]^{\text {vir }}$ can be defined. There are two ingredients:

- The motivic vanishing cycle of Denef and Loeser [8],

- A principal $\mathbb{Z}_{2}$ bundle determined by the choice of orientation. 
A local virtual motive can be associated to the motivic vanishing cycle. After a motivic twist by the principal $\mathbb{Z}_{2}$ bundle, Joyce and collaborators [16] show the local motives glue together.

We review the motivic vanishing cycle following [8]. For our examples, we will only require the case where $f$ is of the form

$$
f=\prod_{i=1}^{n} z_{i}^{n_{i}}
$$

Here $\left(z_{1}, \ldots, z_{n}\right)$ are coordinates in a neighborhood $U$ of the origin in $\mathbb{C}^{n}$. Put $U_{0}=f^{-1}(0)$.

For an index set $I \subset\{1, \ldots, n\}$ define $E_{I}$ by the equations $z_{i}=0$ for all $i \in I$, and define

$$
E_{I}^{\circ}=E_{I}-\cup_{j \notin I} E_{j} .
$$

Let $m_{I}=\operatorname{gcd}\left\{n_{i} \mid i \in I\right\}$ and define $\widetilde{E}_{I} \rightarrow E_{I}^{\circ}$ by

$$
\widetilde{E}_{I}=\left\{(z, w) \in E_{I}^{\circ} \times \mathbb{C} \mid w^{m_{I}}=\prod_{j \notin I} z_{j}^{n_{j}}\right\}
$$

with a natural projection to $E_{I}^{\circ}$.

The group $\mu_{m_{I}}$ of roots of unity acts on $\widetilde{E}_{I}$ by its action on $w$. In fact, $\widetilde{E}_{I}$ is a Galois $\mu_{m_{I}}$-cover of $E_{I}^{\circ}$. Denoting the action by $\rho_{I}$, we obtain an element $\left[\widetilde{E}_{I}, \rho_{I}\right]$ in the ring of equivariant motives over $U$. The motivic nearby cycle of $f$ is

$$
\mathrm{MF}_{U, f}^{\mathrm{mot}}=\sum_{I \neq \emptyset}(1-\mathrm{L})^{|I|-1}\left[\widetilde{E}_{I}, \rho_{I}\right],
$$

where $\mathrm{L}=\left[\mathbb{A}^{1}\right]$ as before.

The motivic vanishing cycle of $f$ is

$$
\mathrm{MF}_{U, f}^{\mathrm{mot}, \phi}=\mathrm{L}^{-\operatorname{dim} U / 2}\left(\left[U_{0}\right]-\mathrm{MF}_{U, f}^{\mathrm{mot}}\right)
$$

We only need the motivic vanishing cycle in three special cases:

- $f=0$

- $f=z_{1}^{2} z_{2}^{2}$

- $f=z_{1}^{2} z_{2}$ 
In case $f=0$, we have $X=U_{0}=U$ is nonsingular, $\mathrm{MF}_{U, f}^{\mathrm{mot}}$ is empty, and so the motivic vanishing cycle is $\mathrm{L}^{-\operatorname{dim} U / 2}[U]$. Here, we match the conventions of Section 6 .

In case $f=z_{1}^{2} z_{2}^{2}$, for every nonempty $I \subset\{1,2\}$, we have $m_{I}=2$ and $\widetilde{E}_{I}$ is a disconnected double cover of $E_{I}^{\circ}$. So each summand in the motivic nearby cycle (7.2) is the product of the respective $E_{I}^{\circ}$ with the absolute equivariant motive

$$
\left[\mu_{2}, \rho\right]
$$

where $\rho$ denotes the action of $\mu_{2}$ on itself. We therefore obtain

$$
\mathrm{MF}_{U, f}^{\mathrm{mot}}=\left[\mu_{2}, \rho\right]\left(\left[E_{1}^{\circ}\right]+\left[E_{2}^{\circ}\right]+(1-\mathrm{L})\left[E_{12}^{\circ}\right]\right) .
$$

Using (2.2), we find the following expression:

$$
\begin{aligned}
\mathrm{MF}_{U, f}^{\mathrm{mot}, \phi} & = \\
& \mathrm{L}^{-\operatorname{dim} U / 2}\left(\mathrm{~L}^{1 / 2}\left(\left[E_{1}^{\circ}\right]+\left[E_{2}^{\circ}\right]\right)+\left(1-\left(1-\mathrm{L}^{1 / 2}\right)(1-\mathrm{L})\right) E_{12}^{\circ}\right)
\end{aligned}
$$

which simplifies to

$$
\mathrm{L}^{-\operatorname{dim} M / 2}\left([M]+\left[E_{12}^{\circ}\right]\left(\mathrm{L}^{1 / 2}-\mathrm{L}\right)\right) .
$$

In case $f=z_{1}^{2} z_{2}$, we have $m_{1}=2$ and $m_{I}=1$ otherwise. Thus,

$$
\mathrm{MF}_{U, f}^{\mathrm{mot}}=\left[\widetilde{E}_{1}, \rho_{1}\right]+\left[E_{2}^{\circ}\right]+(1-\mathrm{L})\left[E_{12}^{\circ}\right]
$$

and

$$
\mathrm{MF}_{U, f}^{\mathrm{mot}, \phi}=\mathrm{L}^{-\operatorname{dim} U / 2}\left(\left[E_{1}^{\circ}\right]-\left[\widetilde{E}_{1}, \rho_{1}\right]+\mathrm{L}\left[E_{12}^{\circ}\right] .\right)
$$

As expected, $E_{2}^{\circ}$ has cancelled out $\left(E_{2}^{\circ}\right.$ is not part of Crit $(f)$ ).

The principal $\mathbb{Z}_{2}$ bundle associated with a choice of orientation is given by the local isomorphisms

$$
\left.\left.\left(K_{M}^{\mathrm{vir}}\right)^{1 / 2}\right|_{\mathrm{Crit}(f)} \simeq K_{U}\right|_{\operatorname{Crit}(f)}
$$

which are square roots of the canonical isomorphism (7.1).

In the case $f=0$ or $f=z_{1}^{2} z_{2}^{2}$, the principal bundle $\mathbb{Z}_{2}$ bundle is trivial in a punctured neighborhood of each $E_{I}^{\circ}$, essentially since there is no ramification in the Galois $\mu_{2}$ covers described above. As we 
shall see, the principal $\mathbb{Z}_{2}$ bundle plays an important role in the case $f=z_{1}^{2} z_{2}$.

Next we globalize the $f=0$ geometry. Suppose $M$ is nonsingular. As a $d$-critical locus, $M$ can be described by a single critical chart $(M, 0)$, so

$$
K_{M}^{\mathrm{vir}} \cong K_{M}^{\otimes 2} .
$$

For the natural choice of orientation $K_{M}$, we have

$$
[M]^{\mathrm{vir}}=\mathrm{L}^{-\operatorname{dim} M / 2}[M],
$$

If there is no 2-torsion in $\operatorname{Pic}(M)$, then $K_{M}$ is the only possible orientation, as will be the case in our example.

In a second geometry which will arise, $M^{\text {red }}$ is a union of two nonsingular components $E_{1}, E_{2}$ meeting transversally along a nonsingular irreducible divisor $E_{12}$. In such case, $M$ must be nonreduced. We will also have $E_{1}-E_{2}$ and $E_{2}-E_{1}$ nonsingular, so nilpotents can occur only along $E_{12}$. We assume the simplest possible scheme structure compatible with the situation: $\left(z_{1}^{2} z_{2}, z_{1} z_{2}^{2}\right)$ in the neighborhood of any point of any $E_{i j}$, where $z_{1}=0$ and $z_{2}=0$ are local equations for $E_{1}$ and $E_{2}$ respectively. In other words, we take

$$
f=z_{1}^{2} z_{2}^{2}
$$

as the superpotential.

Consider the natural isomorphism

$$
\left.K_{M}^{\mathrm{vir}}\right|_{E_{1}^{\circ}} \cong K_{E_{1}^{\circ}}^{\otimes 2}
$$

These bundles extend to respective line bundles $\left.K_{M}^{\mathrm{vir}}\right|_{E_{1}}$ and $K_{E_{1}}^{\otimes 2}$ on $E_{1}$. Direct computation shows the isomorphism vanishes to order 2 along $E_{12}$. So

$$
\left.K_{M}^{\mathrm{vir}}\right|_{E_{1}} \cong K_{E_{1}}^{\otimes 2}\left(-2 E_{12},\right)
$$

with the analogous identification on $E_{2}$.

So there is again a natural orientation $\left(K_{M}^{\mathrm{vir}}\right)^{1 / 2}$ determined by

$$
\left.\left(K_{M}^{\mathrm{vir}}\right)^{1 / 2}\right|_{E_{i}} \cong K_{E_{i}}\left(-E_{12}\right)
$$


for $i=1,2$. If in addition there is no 2-torsion in $\operatorname{Pic}\left(E_{1}\right)$ or $\operatorname{Pic}\left(E_{2}\right)$, then the orientation is unique. Such uniqueness will occur in our example.

With the unique orientation, we have a globalization of (17.3),

$$
[M]^{\mathrm{vir}}=\mathrm{L}^{-\operatorname{dim} M / 2}\left([M]+\left[E_{12}\right]\left(\mathrm{L}^{1 / 2}-\mathrm{L}\right)\right) .
$$

A third geometry will arise: $M^{\text {red }}$ is irreducible, nonsingular, and contains a nonsingular divisor $D \subset M$ precisely along which $M$ is nonreduced. We assume the simplest possible scheme structure compatible with the situation, $\left(z_{1}^{2}, z_{1} z_{2}\right)$ in the neighborhood of any point of $D$, where $z_{1}=0$ is a local equation for $M^{\text {red }}$ and $z_{1}=z_{2}=0$ are local equations for $D$. In other words, we take

$$
f=z_{1}^{2} z_{2}
$$

as the superpotential 13 locally identifying $E_{1}$ with $M^{\text {red }}$ and $E_{12}$ with $D$.

Consider the natural isomorphism

$$
\left.K_{M}^{\mathrm{vir}}\right|_{M^{\mathrm{red}-D}} \cong K_{M^{\mathrm{red}}-D}^{\otimes 2}
$$

These bundles extend to respective line bundles $K_{M}^{\mathrm{vir}}$ and $K_{M^{\text {red }}}^{\otimes 2}$ on $M^{\text {red }}$. Direct computation shows the isomorphism vanishes to order 1 along $D$. So

$$
K_{M}^{\mathrm{vir}} \cong K_{M^{\text {red }}}^{\otimes 2}(-D)
$$

It is apparent that there is no natural orientation $\left(K_{M}^{\mathrm{vir}}\right)^{1 / 2}$ in this general situation.

For the moduli space of stable pairs, we know that $M$ is an oriented $d$-critical locus by general theory. Hence, we conclude $D$ must be even:

$$
\mathcal{O}_{M^{\text {red }}}(D) \simeq L^{\otimes 2}
$$

\footnotetext{
${ }^{13}$ The superpotential $z_{1}^{2} z_{2}$ together with the associated $\mathbb{Z}_{2}$-monodromy was first analyzed in [32, Example 4.5] to calculate nontrivial refined stable pairs invariants of local $\mathbb{P}^{1}$.
} 
for some line bundle $L$ on $M^{\text {red }} 14$ Then, we have an orientation

$$
\left(K_{M}^{\mathrm{vir}}\right)^{1 / 2}=K_{M^{\mathrm{red}}} \otimes L^{-1}
$$

which is the only possibility if $\operatorname{Pic}(M)$ has no 2-torsion.

In the above oriented situation, the principal $\mathbb{Z}_{2}$ bundle of square roots of (7.1) in a critical chart naturally ramifies when extended to $D$. Let

$$
\pi: \widetilde{M} \rightarrow M
$$

be the double cover of $M$ branched along $D$, with the natural involution $\iota$ and ramification divisor $\tilde{D}$. The principal $\mathbb{Z}_{2}$ bundle modifies the local virtual motive $\mathrm{L}^{-\operatorname{dim} M / 2}[M-D]$ of $M-D$ td 15

$$
\mathrm{L}^{-(\operatorname{dim} M+1) / 2}([M-D]-[\widetilde{M}-\widetilde{D}, \iota]) .
$$

Comparison with (7.4) shows how to extend the motive globally. The result is

$$
\mathrm{L}^{-(\operatorname{dim} M+1) / 2}([M-D]-[\widetilde{M}-\widetilde{D}, \iota]+\mathrm{L}[D])
$$

We now compute the virtual Poincaré polynomial of (17.7). To convert a $\mathbb{Z}_{2}$-equivariant motive $[V, \iota]$ to a virtual Poincaré polynomial, decompose $H_{\mathrm{c}}^{*}(V)$ into its even and odd parts under $\iota^{*}$ :

$$
H_{\mathrm{c}}^{*}(V)=H_{\mathrm{c}}^{+}(V) \oplus H_{\mathrm{c}}^{-}(V)
$$

and then take the virtual Poincaré polynomial, which we write as

$$
\widetilde{\mathrm{H}}(V)=\widetilde{\mathrm{H}}_{\mathrm{c}}^{+}(V)+\widetilde{\mathrm{H}}_{\mathrm{c}}^{-}(V)
$$

In our conventions the virtual Poincaré polynomial of $[V, \iota]$ is then

$$
\widetilde{\mathrm{H}}(V, \iota)=\widetilde{\mathrm{H}}_{\mathrm{c}}^{+}(V)-u \widetilde{\mathrm{H}}_{\mathrm{c}}^{-}(V),
$$

\footnotetext{
${ }^{14}$ The same calculation was applied in [16, Example 2.39] to the situation

$$
\left(M^{\mathrm{red}}, D\right)=\left(\mathbb{P}^{1}, p\right)
$$

to show that a certain $d$-critical locus was not orientable since the class of a point is not even in $\operatorname{Pic}\left(\mathbb{P}^{1}\right)$.

${ }^{15}$ In 4 , the $\mathbb{Z}_{2}$ twists are only defined in a quotient of the equivariant motivic ring. We presume the computation holds in the equivariant motivic ring itself if other approaches to virtual motives are followed [23].
} 
see [24, Corollary 7.2]. The minus sign in (7.8) is consistent with the evaluation

$$
\widetilde{\mathrm{H}}\left(\mathrm{L}^{1 / 2}\right)=\widetilde{\mathrm{H}}\left(1-\left[\mu_{2}, \rho\right]\right)=u .
$$

Finally, the virtual Poincaré polynomial of (7.7) is

$$
u^{-(\operatorname{dim} M+1)}\left(u \widetilde{\mathrm{H}}_{\mathrm{c}}^{-}(\widetilde{M})+u^{2} \widetilde{\mathrm{H}}_{c}(D)\right) .
$$

7.2. Poincaré polynomials. Some elucidation of (7.8) is in order here. Let $K_{0}^{\widehat{\mu}}(\mathrm{HS})$ denote the Grothendieck ring of the category of Hodge structures with a $\widehat{\mu}$-action. There is a Hodge characteristic map 24],

$$
\chi_{\mathrm{h}}: K_{\mathrm{var}}^{\widehat{\mu}} \rightarrow K_{0}^{\widehat{\mu}}(\mathrm{HS}),
$$

which can be extended to

$$
\chi_{\mathrm{h}}: K_{\mathrm{var}}^{\widehat{\mu}}\left[\mathrm{L}^{-1}\right] \rightarrow K_{0}^{\widehat{\mu}}(\mathrm{HS})
$$

since $\chi_{\mathrm{h}}(\mathrm{L})$ is invertible. We will define a virtual Poincaré polynomial map

$$
\mathrm{P}: K_{0}^{\widehat{\mu}}(\mathrm{HS}) \rightarrow \mathrm{Z}\left[u, u^{-1}\right]
$$

which then determines a virtual Poincaré polynomial 16 map

$$
\widetilde{\mathrm{H}}=\mathrm{P} \circ \chi_{h}: K_{\mathrm{var}}^{\widehat{\mu}}\left[\mathbb{L}^{-1}\right] \rightarrow \mathrm{Z}\left[u, u^{-1}\right]
$$

The definition of $P$ is chosen so that $\widetilde{H}$ is a ring homomorphism.

For simplicity of exposition, we focus on the special case of $\mu_{2^{-}}$ equivariant Hodge structures (the only case which appears in the examples considered here) and say a few words about how to extend to the general case.

By [24, Cor. 7.2], the motivic convolution product in $K_{\mathrm{var}}^{\widehat{\mu}}$ descends to a product in $K_{0}^{\widehat{\mu}}(\mathrm{HS})$, denoted $*$. Restricting to elements of $K_{0}^{\mu_{2}}(\mathrm{HS})$, the product is shown to satisfy

$$
\begin{aligned}
& \text { (7.11) } H * H^{\prime}= \\
& H^{+} \otimes\left(H^{\prime}\right)^{+}+H^{+} \otimes\left(H^{\prime}\right)^{-}+H^{-} \otimes\left(H^{\prime}\right)^{+}+H^{-} \otimes\left(H^{\prime}\right)^{-}(-1),
\end{aligned}
$$

\footnotetext{
${ }^{16}$ Equivalent definitions have appeared before, see [33, Appendix A.4] and the references there.
} 
where $H^{ \pm}$are the even and odd parts of the $\mu_{2}$-action on $H$ (and similarly for $\left.H^{\prime}\right)$.

Forgetting the $\mu_{2}$ action, we let $\mathrm{Q}(H)$ be the Poincaré polynomial of a Hodge structure $H$. Equation (7.8) can be rephrased as defining $\mathrm{P}$ to be

$$
\mathrm{P}(H)=\mathrm{Q}\left(H^{+}\right)-u \mathrm{Q}\left(H^{-}\right) .
$$

Then (17.11) implies $\mathrm{P}\left(H * H^{\prime}\right)$ is

$\mathrm{Q}\left(H^{+} \otimes\left(H^{\prime}\right)^{+}\right)-u \mathbf{Q}\left(H^{+} \otimes\left(H^{\prime}\right)^{-}\right)-u\left(H^{-} \otimes\left(H^{\prime}\right)^{+}\right)+u^{2} \mathbf{Q}\left(H^{-} \otimes\left(H^{\prime}\right)\right)$

which equals $\mathrm{P}(H) \mathrm{P}\left(H^{\prime}\right)$ as desired.

The full result [24, Cor. 7.2] extends (7.11) to a formula for $*$ valid for any

$$
H, H^{\prime} \in K_{0}^{\widehat{\mu}}(\mathrm{HS}),
$$

expressed in terms of characters of $\widehat{\mu}$. Then $\mathrm{P}(H)$ can be defined by $\mathrm{Q}(H)$ for $H$ a trivial representation of $\widehat{\mu}$ and $-u \mathrm{Q}(H)$ if $H$ transforms by a nontrivial character of $\widehat{\mu}$. The verification of

$$
\mathrm{P}\left(H * H^{\prime}\right)=\mathrm{P}(H) \mathrm{P}\left(H^{\prime}\right)
$$

in the general case is a bit more involved, relying on the precise form of [24. Cor. 7.2] and of the computation of the characters of the cohomology of the Fermat curves used in the definition of motivic convolution [31]. The result from [31] also appears as [24, Lemma 7.1].

7.3. Elliptically fibered K3 surfaces. Let $S$ be an elliptically fibered K3 surface,

$$
\pi: S \rightarrow \mathbb{P}^{1},
$$

with section $s$ and fiber class $f$. No singular point of any fiber lies on $s$. We will compute motivic stable pair invariants in classes

$$
s, f, s+f \in \operatorname{Pic}(S)
$$

with small Euler characteristic following the method of [16]. For our definition in Section 2, we will consider various families

$$
\epsilon: T \rightarrow(\Delta, 0)
$$


depending upon the class.

We start with the fiber class $f \in \operatorname{Pic}(S)$. If $(F, \tau)$ is a stable pair of class $[F]=f$ and $\chi(F)=0$, then $F=\mathcal{O}_{E}$ for some fiber $E$ of $\pi$. Since the fibers are parametrized by $\mathbb{P}^{1}$,

$$
P_{0}(S, f) \simeq \mathbb{P}^{1}
$$

and

$$
\left[P_{0}(S, f)\right]^{\mathrm{vir}}=\mathrm{L}^{-1 / 2}\left[\mathbb{P}^{1}\right] .
$$

Note $\operatorname{Pic}\left(\mathbb{P}^{1}\right)$ has no torsion, so there is no choice in the motivic invariant.

If $\chi(F)=1$, then the $\operatorname{coker}(\tau)$ of the stable pair $(F, \tau)$ is a point, and $P_{1}(S, f) \cong S$, so

$$
\left[P_{1}(S, f)\right]^{\mathrm{vir}}=\mathrm{L}^{-1}[S] .
$$

Again, $\operatorname{Pic}(S)$ has no torsion, so there is no choice in the motivic invariant.

Next consider the section $s \in \operatorname{Pic}(S)$. We see $P_{1}(S, s)$ is a point and

$$
P_{2}(S, s) \cong \mathbb{P}^{1} .
$$

Hence, the coefficient of $v^{s}$ in the motivic partition function $Z^{\text {mot }}$ is

$$
q+\mathrm{L}^{-1 / 2}\left[\mathbb{P}^{1}\right] q^{2}+\ldots .
$$

Since $s, f \in \operatorname{Pic}(S)$ are irreducible classes, for any 1-rigid family $\epsilon$, the above moduli identifications are valid on $T$.

The class $s+f$ is primitive but not irreducible. In the Euler characteristic 0 case,

$$
P_{0}(S, s+f) \cong \mathbb{P}^{1}
$$

is determined by the location of a fiber of $\pi$. Hence,

$$
\left[P_{0}(S, s+f)\right]^{\mathrm{vir}}=\mathrm{L}^{-1 / 2}\left[\mathbb{P}^{1}\right] .
$$

Again, $T$ plays no interesting role.

The more interesting geometry occurs in Euler characteristic 1. The moduli space $P_{1}(T, s+f)$ has two components, $E_{1}$ where the point is on the fiber and $E_{2}$ where the point is on $s$ : 
- $E_{1} \cong S$

- $E_{2} \cong \mathbb{P}^{1} \times \mathbb{P}^{1}$

For $E_{2}$, the first $\mathbb{P}^{1}$ parametrizes the point of the fiber and the second $\mathbb{P}^{1}$ parametrizes the location of the point $\operatorname{coker}(\tau)$ on the section. The two components meet along

$$
E_{1} \cap E_{2} \cong \mathbb{P}^{1}
$$

embedded in $S$ as the section $s$ and in $\mathbb{P}^{1} \times \mathbb{P}^{1}$ as the diagonal.

We assume the local superpotential is the second form discussed in Section 7.1. By (7.5), we have

$$
\left[P_{1}(X, s+f)\right]^{\mathrm{vir}}=\mathrm{L}^{-1}\left(\left[P_{1}(X, s+f)\right]+\left[\mathbb{P}^{1}\right]\left(\mathrm{L}^{1 / 2}-\mathrm{L}\right)\right)
$$

For the classes $s, f, s+f \in \operatorname{Pic}(S)$ to order $q$, the motivic partition function is

$$
\begin{aligned}
\mathrm{Z}^{\mathrm{mot}}=1 & +v^{s}(q+\ldots) \\
& +v^{f}\left(\mathrm{~L}^{-1 / 2}\left[\mathbb{P}^{1}\right]+q \mathrm{~L}^{-1}[S]+\ldots\right) \\
& +v^{s+f}\left(\mathrm{~L}^{-1 / 2}\left[\mathbb{P}^{1}\right]+q \mathrm{~L}^{-1}\left(\left[P_{1}(X, s+f)\right]+\left[\mathbb{P}^{1}\right]\left(\mathrm{L}^{1 / 2}-\mathrm{L}\right)\right)+\ldots\right) \\
& +\ldots .
\end{aligned}
$$

We now calculate the coefficient of $v^{s+f}$ in $\log \left(Z^{\text {mot }}\right)$. The $q^{0}$ coefficient is simply $\mathrm{L}^{-1 / 2}\left[\mathbb{P}^{1}\right]$. The $q$ coefficient is

$$
\begin{aligned}
& \mathrm{L}^{-1}\left(\left[P_{1}(X, s+f)\right]+\left[\mathbb{P}^{1}\right]\left(\mathrm{L}^{1 / 2}-\mathrm{L}\right)\right)-\mathrm{L}^{-1 / 2}\left[\mathbb{P}^{1}\right] \\
& =\mathrm{L}^{-1}\left([S]+\left[\mathbb{P}^{1} \times \mathbb{P}^{1}\right]-\left[\mathbb{P}^{1}\right]+\left[\mathbb{P}^{1}\right]\left(\mathrm{L}^{1 / 2}-\mathrm{L}\right)\right)-\mathrm{L}^{-1 / 2}\left[\mathbb{P}^{1}\right] \\
& \quad=\mathrm{L}^{-1}[S]+\mathrm{L}^{-1}\left[\mathbb{P}^{1}\right]\left(\left[\mathbb{P}^{1}\right]-1+\mathrm{L}^{1 / 2}-\mathrm{L}\right)-\mathrm{L}^{-1 / 2}\left[\mathbb{P}^{1}\right]
\end{aligned}
$$

The last expression is easily simplified to $\mathrm{L}^{-1}[S]$. We conclude the $q^{0}$ and $q^{1}$ coefficients of $v^{s+f}$ in $\log \left(Z^{\text {mot }}\right)$ agree with the $q^{0}$ and $q^{1}$ coefficients of $v^{f}$ in $\log \left(Z^{\mathrm{mot}}\right)$.

The above calculation provides nontrivial evidence for Conjectures $\mathrm{A}$ and $\mathrm{B}$. In fact, if more naive approaches to the motivic theory are taken (for example using the actual moduli spaces or even the Behrend function on the moduli spaces), the agreement we have found fails. We 
have verified the prediction to order $q^{2}$, but we do not include the more involved calculations here.

We next turn to the class $2 f$. We have

$$
P_{0}(S, 2 f) \cong \operatorname{Sym}^{2}\left(\mathbb{P}^{1}\right) \cong \mathbb{P}^{2}
$$

However, in the algebraic twistor family, the scheme structure is not reduced 17 precisely along the diagonal curve $D \subset \mathbb{P}^{2}$, a plane conic. The discussion of Section 7.1 therefore applies. The virtual canonical bundle is

$$
K_{\mathbb{P}^{2}}^{\otimes 2}(-D) \cong \mathcal{O}_{\mathbb{P}^{2}}(-8),
$$

and the algebraic twistor family is uniquely oriented by $\mathcal{O}_{\mathbb{P}^{2}}(-4)$.

The double cover of $\mathbb{P}^{2}$ branched along $D$ is a nonsingular quadric surface $Q$ containing an isomorphic copy $\widetilde{D}$ of $D$. The motive of $D \cong \widetilde{D}$ is $\mathrm{L}+1$, so the motive of $Q-\widetilde{D}$ is $\mathrm{L}^{2}+\mathrm{L}$ and the motive of $\mathbb{P}^{2}-D$ is $\mathrm{L}^{2}$. Therefore the odd part of the motive of $Q-\widetilde{D}$ is L. Hence (7.9) yields

$$
\mathrm{H}_{0,2,0[2]}=\widetilde{\mathrm{H}}\left(\left[P_{0}(X, 2 f)\right]^{\text {vir }}\right)=1+\left(u+u^{-1}\right),
$$

in complete agreement with Conjecture C.

\section{Duality And Noether-Lefschetz theory}

8.1. Heterotic-Type II duality. The Yau-Zaslow conjecture originates in heterotic-Type II duality in 6d, where the heterotic string is compactified on the four torus $T^{4}$ and the Type II string on $K 3$. By the adiabatic argument [35], this can be extended to $4 \mathrm{~d} N=2$ supersymmetric theories, which are obtained from dual pairs of heterotic string compactifications on $K 3 \times T^{2}$ and Type II string compactifications on Calabi-Yau 3-folds $X$. The latter are $K 3$ fibations over $\mathbb{P}^{1}$. This construction of dual pairs requires a match between the vector- and hypermultiplet moduli spaces of the heterotic and the type II compactifications. The heterotic moduli parametrize the metric of $K 3 \times T^{2}$, the bundle data of the heterotic compactification, and the

\footnotetext{
${ }^{17}$ We thank R. Thomas for the verification.
} 
heterotic dilaton $S$, which is in a vector multiplet. In Type IIA compactifications, the complexified Kähler moduli $(t, S)$ of $X$ parametrize the vector multiplet moduli space 18 , which is of complex dimension $h^{11}(X)$. In particular, the heterotic dilaton

$$
S=\frac{4 \pi}{g_{\text {het }}^{2}}+i \theta
$$

is identified with the complexified volume of the base $\mathbb{P}^{1}$.

The simplest example is the STU-model, see [22] for review. Here, the Calabi-Yau 3 -fold $X$ is an elliptic fibration over $\mathbb{P}^{1} \times \mathbb{P}^{1}$ and a $K 3$ fibration over $\mathbb{P}^{1}$ with

$$
h^{11}(X)=3 .
$$

The three vector moduli are identified on the heterotic side with the heterotic dilaton $S$, the complex modulus $T$, and the complexified Kähler modulus $U$ of the heterotic torus $T^{2} 19$.

An impressive consequence of the proposed duality is that a perturbative heterotic one-loop amplitude predicts all higher genus amplitudes

$$
F(\lambda, t)=\sum_{g} \lambda^{2 g-2} F_{g}(t)
$$

of $X$ in the infinite base limit (the dependence on the $K 3$ fiber classes). For the STU model,

$$
\lim _{S \rightarrow \infty} F(\lambda, S, T, U)=F_{\text {het }}^{1-\text { loop }}(\lambda, T, U) .
$$

We will use this relation in the holomorphic limit to extend Conjecture C to a proposal for the refined invariants of the STU model.

\subsection{Refined Noether-Lefschetz theory.}

\footnotetext{
${ }^{18}$ The complex moduli of $X$ together with the Ramond fields and the type II dilaton parametrize the hypermultiplet moduli space, which is of quaternionic dimension $h^{21}(X)+1$. This makes the duality much richer, but we focus on the vector moduli.

${ }^{19} \mathrm{As}^{21}(X)=243$, the heterotic hypermultiplet moduli space is of quaternionic dimension 244 .
} 
8.2.1. Overview. We pass now from the string point of view to the more precise mathematical perspective advanced in [25, 30] as NoetherLefschetz correspondences. For our study of refined invariants, the Noether-Lefschetz numbers of [25, 30] also require refinement.

8.2.2. $\Lambda$-polarization. Following the notation of [30, Section 1.1], let $\Lambda$ be a fixed rank $r$ primitive 20 sublattice

$$
\Lambda \subset U \oplus U \oplus U \oplus E_{8}(-1) \oplus E_{8}(-1)
$$

with signature $(1, r-1)$, and let $v_{1}, \ldots, v_{r} \in \Lambda$ be an integral basis. The discriminant is

$$
\Delta(\Lambda)=(-1)^{r-1} \operatorname{det}\left(\begin{array}{ccc}
\left\langle v_{1}, v_{1}\right\rangle & \cdots & \left\langle v_{1}, v_{r}\right\rangle \\
\vdots & \ddots & \vdots \\
\left\langle v_{r}, v_{1}\right\rangle & \cdots & \left\langle v_{r}, v_{r}\right\rangle
\end{array}\right) .
$$

The sign is chosen so $\Delta(\Lambda)>0$.

A $\Lambda$-polarization of a $K 3$ surface $S$ is a primitive embedding

$$
j: \Lambda \rightarrow \operatorname{Pic}(S)
$$

satisfying two properties:

(i) the lattice pairs $\Lambda \subset U^{3} \oplus E_{8}(-1)^{2}$ and $\Lambda \subset H^{2}(S, \mathbb{Z})$ are isomorphic via an isometry which restricts to the identity on $\Lambda$,

(ii) $\operatorname{Im}(j)$ contains a quasi-polarization.

By (ii), every $\Lambda$-polarized $K 3$ surface is algebraic.

The period domain $M$ of Hodge structures of type $(1,20,1)$ on the lattice $U^{3} \oplus E_{8}(-1)^{2}$ is an analytic open set of the 20-dimensional nonsingular isotropic quadric $Q$,

$$
M \subset Q \subset \mathbb{P}\left(\left(U^{3} \oplus E_{8}(-1)^{2}\right) \otimes_{\mathbb{Z}} \mathbb{C}\right) .
$$

Let $M_{\Lambda} \subset M$ be the locus of vectors orthogonal to the entire sublattice $\Lambda \subset U^{3} \oplus E_{8}(-1)^{2}$.

Let $\Gamma$ be the isometry group of the lattice $U^{3} \oplus E_{8}(-1)^{2}$, and let

$$
\Gamma_{\Lambda} \subset \Gamma
$$

\footnotetext{
${ }^{20} \mathrm{~A}$ sublattice is primitive if the quotient is torsion free.
} 
be the subgroup restricting to the identity on $\Lambda$. By global Torelli, the moduli space $\mathcal{M}_{\Lambda}$ of $\Lambda$-polarized $K 3$ surfaces is the quotient

$$
\mathcal{M}_{\Lambda}=M_{\Lambda} / \Gamma_{\Lambda}
$$

We refer the reader to [10] for a detailed discussion.

8.2.3. Noether-Lefschetz divisors. Let $(\mathbb{L}, \iota)$ be a rank $r+1$ lattice $\mathbb{L}$ with an even symmetric bilinear form $\langle$,$\rangle and a primitive embedding$

$$
\iota: \Lambda \rightarrow \mathbb{L}
$$

Two data sets $(\mathbb{L}, \iota)$ and $\left(\mathbb{L}^{\prime}, \iota^{\prime}\right)$ are isomorphic if and only if there exist an isometry relating $\mathbb{L}$ and $\mathbb{L}^{\prime}$ which takes $\iota$ to $\iota^{\prime}$. The first invariant of the data $(\mathbb{L}, \iota)$ is the discriminant $\Delta \in \mathbb{Z}$ of $\mathbb{L}$.

An additional invariant of $(\mathbb{L}, \iota)$ can be obtained by considering any vector $v \in \mathbb{L}$ for which 21

$$
\mathbb{L}=\iota(\Lambda) \oplus \mathbb{Z} v .
$$

The pairing

$$
\langle v, \cdot\rangle: \Lambda \rightarrow \mathbb{Z}
$$

determines an element of $\delta_{v} \in \Lambda^{*}$. Let $G=\Lambda^{*} / \Lambda$ be the quotient defined via the injection $\Lambda \rightarrow \Lambda^{*}$ obtained from the pairing $\langle$,$\rangle on \Lambda$. The group $G$ is abelian of order given by the discriminant $|\Delta(\Lambda)|$. The image

$$
\delta \in G / \pm
$$

of $\delta_{v}$ is easily seen to be independent of $v$ satisfying (8.2). The invariant $\delta$ is the coset of $(\mathbb{L}, \iota)$

By elementary arguments, two data sets $(\mathbb{L}, \iota)$ and $\left(\mathbb{L}^{\prime}, \iota^{\prime}\right)$ of rank $r+1$ are isomorphic if and only if the discriminants and cosets are equal.

\footnotetext{
${ }^{21}$ Here, $\oplus$ is used just for the additive structure (not orthogonal direct sum).
} 
Let $v_{1}, \ldots, v_{r}$ be an integral basis of $\Lambda$ as before. The pairing of $\mathbb{L}$ with respect to an extended basis $v_{1}, \ldots, v_{r}, v$ is encoded in the matrix

$$
\mathbb{L}_{h, d_{1}, \ldots, d_{r}}=\left(\begin{array}{cccc}
\left\langle v_{1}, v_{1}\right\rangle & \cdots & \left\langle v_{1}, v_{r}\right\rangle & d_{1} \\
\vdots & \ddots & \vdots & \vdots \\
\left\langle v_{r}, v_{1}\right\rangle & \cdots & \left\langle v_{r}, v_{r}\right\rangle & d_{r} \\
d_{1} & \cdots & d_{r} & 2 h-2
\end{array}\right) .
$$

The discriminant is

$$
\Delta\left(h, d_{1}, \ldots, d_{r}\right)=(-1)^{r} \operatorname{det}\left(\mathbb{L}_{h, d_{1}, \ldots, d_{r}}\right) .
$$

The coset $\delta\left(h, d_{1}, \ldots, d_{r}\right)$ is represented by the functional

$$
v_{i} \mapsto d_{i}
$$

The Noether-Lefschetz divisor $P_{\Delta, \delta} \subset \mathcal{M}_{\Lambda}$ is the closure of the locus of $\Lambda$-polarized $K 3$ surfaces $S$ for which $(\operatorname{Pic}(S), j)$ has rank $r+1$, discriminant $\Delta$, and coset $\delta$. By the Hodge index theorem $22, P_{\Delta, \delta}$ is empty unless $\Delta>0$. By definition, $P_{\Delta, \delta}$ is a reduced subscheme.

Let $h, d_{1}, \ldots, d_{r}$ determine a positive discriminant

$$
\Delta\left(h, d_{1}, \ldots, d_{r}\right)>0
$$

The Noether-Lefschetz divisor $D_{h,\left(d_{1}, \ldots, d_{r}\right)} \subset \mathcal{M}_{\Lambda}$ is defined by the weighted sum

$$
D_{h,\left(d_{1}, \ldots, d_{r}\right)}=\sum_{\Delta, \delta} m\left(h, d_{1}, \ldots, d_{r} \mid \Delta, \delta\right) \cdot\left[P_{\Delta, \delta}\right]
$$

where the multiplicity $m\left(h, d_{1}, \ldots, d_{r} \mid \Delta, \delta\right)$ is the number of elements $\beta$ of the lattice $(\mathbb{L}, \iota)$ of type $(\Delta, \delta)$ satisfying

$$
\langle\beta, \beta\rangle=2 h-2, \quad\left\langle\beta, v_{i}\right\rangle=d_{i} .
$$

If the multiplicity is nonzero, then $\Delta \mid \Delta\left(h, d_{1}, \ldots, d_{r}\right)$ so only finitely many divisors appear in the above sum.

\footnotetext{
${ }^{22}$ The intersection form on $\operatorname{Pic}(S)$ is nondegenerate for an algebraic $K 3$ surface. Hence, a rank $r+1$ sublattice of $\operatorname{Pic}(S)$ which contains a quasi-polarization must have signature $(1, r)$ by the Hodge index theorem.
} 
8.2.4. Refined Noether-Lefschetz numbers. Let $X$ be a nonsingular projective Calabi-Yau 3-fold fibered in $K 3$ surfaces,

$$
\pi: X \rightarrow \mathbb{P}^{1} .
$$

Let $L_{1}, \ldots, L_{r} \in \operatorname{Pic}(X)$ determine a 1 -parameter family of $\Lambda$-polarized $K 3$ surfaces,

$$
\left(X, L_{1}, \ldots, L_{r}, \pi\right) .
$$

The 1-parameter family determines a morphism

$$
\iota: \mathbb{P}^{1} \rightarrow \mathcal{M}_{\Lambda} .
$$

The Noether-Lefschetz number $\mathrm{NL}_{h, d_{1}, \ldots, d_{r}}^{\pi}$ is defined [25, 30] by the following conditions:

- if $\Delta\left(h, d_{1}, \ldots, d_{r}\right)<0$, then $\mathrm{NL}_{h, d_{1}, \ldots, d_{r}}^{\pi}=0$,

- if $\Delta\left(h, d_{1}, \ldots, d_{r}\right)=0$, then $\mathrm{NL}_{h, d_{1}, \ldots, d_{r}}^{\pi}=-2$,

- if $\Delta\left(h, d_{1}, \ldots, d_{r}\right)>0$, the Noether-Lefschetz number is defined by the classical intersection product

$$
\mathrm{NL}_{h,\left(d_{1}, \ldots, d_{r}\right)}^{\pi}=\int_{\mathbb{P}^{1}} \iota_{\pi}^{*}\left[D_{h,\left(d_{1}, \ldots, d_{r}\right)}\right] .
$$

Our refinements of $\mathrm{NL}_{h,\left(d_{1}, \ldots, d_{r}\right)}^{\pi}$ will not be numbers, but rather representation $\$ 23$ of $S U(2) \times S U(2)$ lying in the space

$$
\mathbb{Z}_{\geq 0}[0,0] \oplus \mathbb{Z}_{\geq 0}\left[0, \frac{1}{2}\right] .
$$

The first refinement is defined by

$$
\mathrm{RNL}_{h, d_{1}, \ldots, d_{r}}^{\pi, \circ}=\mathrm{NL}_{h, d_{1}, \ldots, d_{r}}^{\pi}[0,0]
$$

and carries no more data than the Noether-Lefschetz number.

The definition of the second refinement $\mathrm{RNL}_{h, d_{1}, \ldots, d_{r}}^{\pi, \diamond}$ is more subtle. Again, we consider three cases based upon the discriminant:

- if $\Delta\left(h, d_{1}, \ldots, d_{r}\right)<0$, then $\mathrm{RNL}_{h, d_{1}, \ldots, d_{r}}^{\pi, \diamond}=0$,

- if $\Delta\left(h, d_{1}, \ldots, d_{r}\right)=0$, then $\mathrm{RNL}_{h, d_{1}, \ldots, d_{r}}^{\pi, \diamond}=\left[0, \frac{1}{2}\right]$.

\footnotetext{
${ }^{23}$ As before, We denote the irreducible representations of $S U(2) \times S U(2)$ by pairs $\left[j_{L}, j_{R}\right]$ where $j_{L}, j_{R} \in \frac{1}{2} \mathbb{Z}_{\geq 0}$.
} 
If $\Delta\left(h, d_{1}, \ldots, d_{r}\right)>0$, we divide the effective sum (8.3) defining $D_{h,\left(d_{1}, \ldots, d_{r}\right)}$ into two parts

$$
D_{h,\left(d_{1}, \ldots, d_{r}\right)}=S_{\iota}+T_{\iota}
$$

where $S_{\iota}$ is the sum of the divisors on the right side of (8.3) not containing $\iota\left(\mathbb{P}^{1}\right)$ and $T_{\iota}$ is the sum of such divisors containing $\iota\left(\mathbb{P}^{1}\right)$. The final case of the definition is:

- if $\Delta\left(h, d_{1}, \ldots, d_{r}\right)>0$, then

$$
\mathrm{RNL}_{h, d_{1}, \ldots, d_{r}}^{\pi, \diamond}=\int_{\mathbb{P} 1} \iota_{\pi}^{*} S_{\iota} \cdot[0,0]-\frac{1}{2} \int_{\mathbb{P} 1} \iota_{\pi}^{*} T_{\iota} \cdot\left[0, \frac{1}{2}\right] .
$$

The motivation of the second refinement is to record the geometric components of the Noether-Lefschetz locus over the base $\mathbb{P}^{1}$. Such loci here are unions of points and lines - the points correspond to the representation $[0,0]$ and the lines to the representation $\left[0, \frac{1}{2}\right]$.

\subsection{Refined Pairs/Noether-Lefschetz correspondence. We pre-} dict a refined Pairs/Noether-Lefschetz correspondence which intertwines three theories associated to the 1-parameter family

$$
\pi: X \rightarrow \mathbb{P}^{1}
$$

of $\Lambda$-polarized $K 3$ surfaces with Calabi-Yau total space:

(i) the refined Noether-Lefschetz theory of $\pi$,

(ii) the refined Gopakumar-Vafa invariants of $X$ in fiber classes,

(iii) the refined invariants $\mathrm{R}_{j_{L}, j_{R}}^{h}$ of the $K 3$ fibers.

Let $\mathrm{R}_{j_{L}, j_{R},\left(d_{1}, \ldots, d_{r}\right)}^{X}$ denote the refined Gopakumar-Vafa invariants of [7] defined via the stable pairs moduli spaces of $X$ for $\pi$-vertical curve classes of degrees $d_{1}, \ldots, d_{r}$ with respect to line bundles $L_{1}, \ldots, L_{r}$ corresponding to a basis of $\Lambda$. An $r$-tuple $\left(d_{1}, \ldots, d_{r}\right)$ is positive if the associated degree with respect to a quasi-polarization $\lambda^{\pi} \in \Lambda$ is positive.

For our proposed $\mathrm{R} / \mathrm{NL}$ refined correspondence, the $K 3$ invariant $\mathrm{R}_{j_{L}, j_{R}}^{h}$ must be divided into two parts,

$$
\mathrm{R}_{j_{L}, j_{R}}^{h}=\mathrm{R}_{\substack{j_{L}, j_{R} \\ 34}}^{h, \circ}+\mathrm{R}_{j_{L}, j_{R}}^{h,}
$$


Speculation [Refined $\mathrm{P} / \mathrm{NL}$ correspondence]. A 1-parameter family of $\Lambda$-polarized $K 3$ surfaces

$$
\pi: X \rightarrow \mathbb{P}^{1}
$$

with Calabi-Yau total space determines a division (8.4) satisfying the following property. For degrees $\left(d_{1}, \ldots, d_{r}\right)$ positive with respect to the quasi-polarization $\lambda^{\pi}$,

$$
\begin{aligned}
\sum_{j_{L}, j_{R}} \mathrm{~N}_{j_{L}, j_{R}}^{X,\left(d_{1}, \ldots, d_{r}\right)}\left[j_{L}, j_{R}\right]= & \sum_{j_{L}, j_{R}} \sum_{h=0}^{\infty} \mathrm{R}_{j_{L}, j_{R}}^{h, \circ}\left[j_{L}, j_{R}\right] \otimes \mathrm{RNL}_{h,\left(d_{1}, \ldots, d_{r}\right)}^{\pi, \circ} \\
& +\sum_{j_{L}, j_{R}} \sum_{h=0}^{\infty} \mathrm{R}_{j_{L}, j_{R}}^{h,}\left[j_{L}, j_{R}\right] \otimes \mathrm{RNL}_{h,\left(d_{1}, \ldots, d_{r}\right)}^{\pi, \diamond}
\end{aligned}
$$

By vanishing properties of the Noether-Lefschetz numbers, the above summations over $h$ are finite for given $\left(d_{1}, \ldots, d_{r}\right)$. We expect the counts $\mathrm{R}_{j_{L}, j_{R},\left(d_{1}, \ldots, d_{r}\right)}$ to be invariant under deformations of $X$ as a family of $\Lambda$-polarized $K 3$ surfaces.

8.4. STU example. For the STU model, we have a precise conjecture for the division (8.4) of $\mathrm{R}_{j_{L}, j_{R}}^{h}$ which is consistent with several basic calculations.

We follow the STU conventions of [22] with the lattice

$$
\Lambda=\left(\begin{array}{ll}
0 & 1 \\
1 & 0
\end{array}\right) \text {. }
$$

The Noether-Lefschetz numbers for the STU model

$$
\pi: X \rightarrow \mathbb{P}^{1}
$$

are determined in 22 to be

$$
\mathrm{NL}_{h,\left(d_{1}, d_{2}\right)}^{\pi}=\operatorname{Coeff}_{q^{1+d_{1} d_{2}-h}}\left(-2 E_{4}(q) E_{6}(q)\right)
$$

where $E_{4}$ and $E_{6}$ are the Eisenstein series,

$$
-2 E_{4}(q) E_{6}(q)=-2+528 q+270864 q^{2}+10393152 q^{3}+\ldots .
$$

The refinement is easily seen to be given by

$$
\mathrm{RNL}_{h,\left(d_{1}, d_{2}\right)}^{\pi, \diamond}=\left[0, \frac{1}{2}\right]
$$


if $1+d_{1} d_{2}-h=0$ and

$$
\mathrm{RNL}_{h,\left(d_{1}, d_{2}\right)}^{\pi, \diamond}=\mathrm{NL}_{h,\left(d_{1}, d_{2}\right)}^{\pi} \cdot[0,0]
$$

otherwise. The Betti number of $X$ are

$$
u^{-3}[X]=u^{-3}+3 u^{-1}+488+3 u+u^{3} .
$$

We define $\mathrm{R}_{j_{L}, j_{R}}^{h, \diamond}$ for the STU model by a formula parallel to (5.1) but using only part of the generating series of Hodge numbers of the Hilbert schemes of points of $K 3$ surfaces:

$$
\begin{aligned}
\sum_{h=0}^{\infty} & \sum_{j_{L}} \sum_{j_{R}} \mathrm{R}_{j_{L}, j_{R}}^{h, \diamond}\left[j_{L}\right]_{y}\left[j_{R}\right]_{u} q^{h}= \\
& \prod_{n=1}^{\infty} \frac{1}{\left(1-u^{-1} y^{-1} q^{n}\right)\left(1-u^{-1} y q^{n}\right)\left(1-u y^{-1} q^{n}\right)\left(1-u y q^{n}\right)} .
\end{aligned}
$$

\begin{tabular}{|c|c|}
\hline $\mathrm{R}_{\frac{i}{2}, \frac{j}{2}}^{0, \diamond}$ & $\mathrm{i}=0$ \\
\hline $\mathrm{j}=0$ & 1 \\
\hline
\end{tabular}

\begin{tabular}{|c|ll|}
\hline $\mathrm{R}_{\frac{i}{2}, \frac{j}{2}}^{1, \diamond}$ & $\mathrm{j}=0$ & 1 \\
\hline $\mathrm{i}=0$ & & \\
1 & & 1 \\
\hline
\end{tabular}

\begin{tabular}{|c|ccc|}
\hline $\mathrm{R}_{\frac{i}{2}, \frac{j}{2}}^{2, \diamond}$ & $\mathrm{j}=0$ & 1 & 2 \\
\hline $\mathrm{i}=0$ & 1 & & \\
1 & & 1 & \\
2 & & & 1 \\
\hline
\end{tabular}

\begin{tabular}{|c|cccc|}
\hline $\mathrm{R}_{\frac{i}{2}, \frac{j}{2}}^{3, \diamond}$ & $\mathrm{j}=0$ & 1 & 2 & 3 \\
\hline $\mathrm{i}=0$ & 1 & & 1 & \\
1 & & 2 & & \\
2 & 1 & & 1 & \\
3 & & & & 1 \\
\hline
\end{tabular}

\begin{tabular}{|c|ccccc|}
\hline $\mathrm{R}_{\frac{i}{2}, \frac{j}{2}}^{4,}$ & $\mathrm{j}=0$ & 1 & 2 & 3 & 4 \\
\hline $\mathrm{i}=0$ & 3 & & 1 & & \\
1 & & 3 & & 1 & \\
2 & 1 & & 3 & & \\
3 & & 1 & & 1 & \\
4 & & & & & 1 \\
\hline
\end{tabular}

TABLE 3. All nonvanishing $\mathrm{R}_{j_{L}, j_{R}}^{h, \diamond}$ for $h \leq 4$

We expect $\mathrm{R}_{j_{L}, j_{R}}^{h, \diamond}$ to always be nonnegative and bounded by $\mathrm{R}_{j_{L}, j_{R}}^{h}$. Then, $\mathrm{R}_{j_{L}, j_{R}}^{h, \circ}$ is uniquely defined by equations (5.1), (8.4), and (8.5). 
Conjecture D. A refined P/NL correspondence holds for fiber classes of the STU model:

$$
\begin{aligned}
\sum_{j_{L}, j_{R}} \mathrm{~N}_{j_{L}, j_{R}}^{S T U,\left(d_{1}, d_{2}\right)}\left[j_{L}, j_{R}\right]= & \sum_{j_{L}, j_{R}} \sum_{h=0}^{\infty} \mathrm{R}_{j_{L}, j_{R}}^{h, \circ}\left[j_{L}, j_{R}\right] \otimes \mathrm{RNL}_{h,\left(d_{1}, d_{2}\right)}^{\pi, \circ} \\
& +\sum_{j_{L}, j_{R}} \sum_{h=0}^{\infty} \mathrm{R}_{j_{L}, j_{R}}^{h, \diamond}\left[j_{L}, j_{R}\right] \otimes \mathrm{RNL}_{h,\left(d_{1}, d_{2}\right)}^{\pi, \diamond}
\end{aligned}
$$

for degrees $\left(d_{1}, d_{2}\right)$ positive with respect to the quasi-polarization.

Conjectures $\mathrm{C}$ and $\mathrm{D}$ together predict the refined invariants of the STU model in fiber classes. Let $\left(d_{1}, d_{2}\right)=(0,1)$ be the fiber class of the elliptic fibration

$$
\mu: X \rightarrow \mathbb{P}^{1} \times \mathbb{P}^{1} .
$$

For refined invariants of the STU model in class $(0,1)$, the conjectures predict:

$$
488[0,0]+\left[\frac{1}{2}, 0\right]+\left[\frac{1}{2}, 1\right] .
$$

After expanding formula (8.1) of [7] with the refined invariants (8.6) for the STU model $X$ in class $\left(d_{1}, d_{2}\right)=(0,1)$, we obtain predictions for the Betti realizations of the following stable pairs motives:

$$
\begin{aligned}
& u^{-2}\left[P_{0}(X,(0,1))\right]=u^{-2}+2+u^{2}, \\
& u^{-3}\left[P_{1}(X,(0,1))\right]=u^{-3}+3 u^{-1}+488+3 u+u^{3} .
\end{aligned}
$$

The above predictions exactly match the expected geometry

$$
\begin{aligned}
& P_{0}(X,(0,1)) \cong \mathbb{P}^{1} \times \mathbb{P}^{1}, \\
& P_{1}(X,(0,1)) \cong X .
\end{aligned}
$$

In fact, the predictions for the fiber class $(0,1)$ case match for the moduli spaces $P_{m}(X,(0,1))$ of stable pairs for all Euler characteristics $m$.

Conjecture D proposes an exact solution for the Betti realization of the stable pairs motivic invariants for the STU model $X$ in fiber classes. Further values of the refined invariants for the STU model are given below. 
Checking the above prediction for $P_{0}(X,(n, 1))$ is easy for all $n \geq 0$. Further checks in the case $\left(d_{1}, d_{2}\right)=(1,1)$ have been completed (and match Conjecture D). Determining the moduli space and the superpotential becomes harder as the Euler characteristic and the curve class increase.

\begin{tabular}{|c|ccc|}
\hline $\mathrm{N}_{\frac{i}{2}, \frac{j}{2}}^{(0,1)}$ & $\mathrm{j}=0$ & 1 & 2 \\
\hline $\mathrm{i}=0$ & 488 & \\
1 & 1 & 1 \\
\hline
\end{tabular} \begin{tabular}{|c|c|ccc|}
$\mathrm{N}_{\frac{i}{2}, \frac{j}{2}}^{(1,1)}$ & $\mathrm{j}=0$ & 1 & 2 & 3 \\
\hline $\mathrm{i}=0$ & 280964 & 1 & & \\
1 & 1 & 488 & 1 & \\
2 & & 1 & 1 \\
\hline
\end{tabular}

\begin{tabular}{|c|ccccc|}
\hline $\mathrm{N}_{\frac{i}{2}, \frac{j}{2}}^{(2,1)}$ & $\mathrm{j}=0$ & 1 & 2 & 3 & 4 \\
\hline $\mathrm{i}=0$ & 15928440 & 2 & & 1 & \\
1 & 2 & 281452 & 2 & & \\
2 & & 2 & 488 & 1 & \\
3 & & & 1 & & 1 \\
\hline
\end{tabular}

\begin{tabular}{|c|cccccc|}
\hline $\mathrm{N}_{\frac{i}{2}, \frac{j}{2}}^{(3,1)}$ & $\mathrm{j}=0$ & 1 & 2 & 3 & 4 & 5 \\
\hline $\mathrm{i}=0$ & 410133618 & 4 & 488 & 1 & & \\
1 & 3 & 16209892 & 4 & & 1 & \\
2 & 488 & 4 & 281452 & 3 & & \\
3 & 1 & & 2 & 488 & 1 & \\
4 & & & & 1 & & 1 \\
\hline
\end{tabular}

TABLE 4. Refined invariants for the STU model in fiber classes

\section{Summary}

Our goal in the paper is to present a conjectural framework for the evaluation of the motivic stable pairs invariants of $K 3$ surfaces in all curve classes. The first step is a definition using suitably NoetherLefschetz transverse algebraic families. Conjectures A and B predict a deformation invariance for the associated Betti realization. These new Betti properties go beyond the older numerical invariance. Conjecture $\mathrm{C}$ then reduces the entire theory to the primitive (and irreducible) case which is determined by the Kawai-Yoshioka calculation. 
In Conjecture D, we propose a refined Pairs/Noether-Lefschetz correspondence for the STU model $X$. Together, Conjectures A-D provide a rich non-toric setting where motivic invariants are well-behaved.

For Calabi-Yau 3-folds, there are three basic approaches to curve counting (along with several variations):

(i) the Gromov-Witten theory of stable maps,

(ii) the Donaldson-Thomas theory of stable pairs,

(iii) the Gopakumar-Vafa theory of stable sheaves with $\chi=1$.

While (i) and (ii) are foundationally well settled and proven equivalent in many cases, the precise formulation of (iii) is not yet clear (and the equivalence is not understood). As we have already mentioned, for a motivic theory, only the sheaf theoretic approaches (ii) and (iii) are at present possible.

When the moduli space of stable sheaves with 1-dimensional support and Euler characteristic 1 is nonsingular, approach (iii) yields a clear proposal. Nonsingularity of the moduli spaces is certainly rare. However, in the Appendix by R. Thomas, nonsingularity is proven for the stable sheaves associated to $K 3$ surfaces in algebraic families transverse to the relevant Noether-Lefschetz divisors. Nonsingularity is proven for all (even imprimitive) curve classes. The outcome provides a direct approach to the GV motivic theory of $K 3$ surfaces which matches precisely with our stable pair predictions. In particular, the GV approach predicts the divisibility invariance of the original KKV formula.

A path proving Conjectures $\mathrm{A}, \mathrm{B}$, and $\mathrm{C}$ is to start with the $\mathrm{GV}$ moduli spaces as discussed in the Appendix and wall-cross to stable pairs 24 Various difficulties aries: the most signficant of which may be the inability to achieve Euler characteristic 1 by twisting by line bundles in the imprimitive case. Nevertheless, proving a Pairs/GV

\footnotetext{
${ }^{24}$ See [34] where exactly the same strategy is applied to study the Euler characteristics of moduli spaces of stable pairs on $S \times \mathbb{C}$. The difficulties left open [34] arise here as well.
} 
correspondence appears the most promising approach to Conjectures $\mathrm{A}, \mathrm{B}$, and $\mathrm{C}$ at the moment. 


\section{Appendix A. ReFined KKV from Refined Gopakumar-VAFA}

by R. P. Thomas

Gopakumar and Vafa have suggested a conjectural approach to defining BPS numbers via moduli of sheaves. S. Katz [18] proposed using stable sheaves of Euler characteristic 1. The papers [14, 21] make BPS and motivic predictions of GV invariants via the geometry of such stable sheaves. We show here for fibre classes of sufficiently NoetherLefschetz transverse $K 3$-fibred 3-folds, the proposals of [14, 21] work perfectly for both the BPS numbers and their refinements: we recover the generating series of Hodge numbers of Hilbert schemes of points on $K 3$ surfaces (5.1) as considered in the current paper.

By a flat family of stable sheaves on the fibres of a projective family

$$
\pi: \mathcal{Y} \rightarrow B
$$

we mean a coherent sheaf $\mathcal{F}$ on $\mathcal{Y}$ which is flat over $B$, such that $\mathcal{F}_{b}$ is stable on $\mathcal{Y}_{b}$ for any closed point $b \in B$. We will need the following standard result.

Lemma A.1. Let $\mathcal{F}$ be a flat family of stable sheaves on the fibres of $\pi: \mathcal{Y} \rightarrow B$. Then $\pi_{*} \mathscr{H}$ om $(\mathcal{F}, \mathcal{F})=\mathcal{O}_{B}$

Proof. Stable sheaves are simple, so

$$
\operatorname{Hom}\left(\mathcal{F}_{b}, \mathcal{F}_{b}\right) / \mathbb{C} \cdot \mathrm{id}=0
$$

for all closed point $b \in B$. It follows by base change that

$$
\pi_{*} \mathscr{H} o m(\mathcal{F}, \mathcal{F}) / \mathcal{O}_{B} \cdot \text { id }=0,
$$

which gives the result.

Now fix $T \stackrel{\pi}{\rightarrow}(\Delta, 0)$, a $K 3$-fibred 3 -fold with central fibre $\pi^{-1}(0)$ given by

$$
\iota: S \hookrightarrow T
$$

Suppose the curve $\Delta$ is transverse to $N L_{\beta}$ for some fixed

$$
\beta \in H^{2}(S, \mathbb{Z}) \cap H^{1,1}(S) .
$$


By shrinking $\Delta$ if necessary, we may assume that $\pi$ is smooth and intersects $N L_{\beta}$ only in 0 .

Consider the moduli space $\mathcal{M}_{1, \iota * \beta}(T)$ of stable 25 dimension 1 sheaves on $T$ with fundamental class $\iota_{*} \beta$ and holomorphic Euler characteristic $\chi=1$. The last condition ensures that no semistable sheaves exist, so $\mathcal{M}_{1, \iota_{*} \beta}(T)$ is projective. By simplicity, any stable sheaf must be scheme theoretically supported on a single fibre of $\pi$. By the Noether-Lefschetz condition, there is an open and closed component

$$
\mathcal{M}^{\star} \subset \mathcal{M}_{1, \iota_{*} \beta}(T)
$$

of sheaves with scheme theoretic support on $S$. Hence, the obvious map

$$
\iota_{*}: \mathcal{M}_{1, \beta}(S) \longleftrightarrow \mathcal{M}^{\star}
$$

is a set-theoretic bijection.

Proposition A.3. The map (A.2) is an isomorphism of schemes.

Proof. The statement is local, so we may work on a Zariski open subset $U \subset \mathcal{M}$. Shrinking $U$ if necessary, we may assume there is a universal sheaf $\mathcal{F}$ on $U \times T$. We must show that $\mathcal{F}$ is the push forward of a sheaf on $U \times S$. The classifying map of the resulting flat family of stable sheaves on $S$ will define the inverse map to (A.2).

Consider the composition

$$
\Gamma\left(\mathcal{O}_{\Delta \times U}\right) \stackrel{p^{*}}{\longrightarrow} \Gamma\left(\mathcal{O}_{T \times U}\right) \stackrel{\text { id }}{\longrightarrow} \operatorname{Hom}(\mathcal{F}, \mathcal{F}) \cong \Gamma\left(\mathcal{O}_{U}\right),
$$

where the final isomorphism is given by Lemma A.1. The map is $\Gamma\left(\mathcal{O}_{U}\right)$-linear so defines a $U$-point of $\Delta \times U$. Its ideal is the kernel of the above composition, and by its definition this ideal annihilates $\mathcal{F}$.

Thus we get a map

$$
f: U \longrightarrow \Delta
$$

such that $\mathcal{F}$ is the pushforward of a sheaf $F$ on $T \times{ }_{\Delta} U \subset T \times U$. Since $\mathcal{F}$ is flat over $U$, so is $F$.

\footnotetext{
${ }^{25}$ Stability is taken with respect to a fixed polarization of $T$.
} 
On closed points $f$ is the constant map to $0 \in \Delta$. To prove $f$ is a constant map of schemes, we may replace $U$ by the formal neighbourhood of any closed point. The result will follow below from Hodge theory and the Noether-Lefschetz transversality condition.

We have shown that $F$ is a flat family of stable sheaves on the smooth family

$$
T \times{ }_{\Delta} U \stackrel{\pi}{\longrightarrow} U
$$

of $K 3$ surfaces over $U$. The fundamental class of $F$ lies in

$$
F^{1} H_{d R}^{2}\left(T \times_{\Delta} U / U\right),
$$

the part of the Hodge filtration defined by $\Omega_{\bar{T} \times_{\Delta} U / U}^{\geq 1}$.

Via the isomorphism [3, Proposition 3.8]

$$
H_{d R}^{2}\left(T \times_{\Delta} U / U\right) \cong H^{2}(S, \mathbb{C}) \otimes_{\Delta} \mathcal{O}_{U}
$$

the class of $F$ is $\beta \otimes 1$. Since it lies in $F^{1}$, its projection

$$
[\beta \otimes 1]^{0,2} \in H_{d R}^{2}\left(T \times_{\Delta} U / U\right) / F^{1} H_{d R}^{2}\left(T \times_{\Delta} U / U\right)
$$

vanishes identically. This class is the pull back via $f$ of the analogous class

$$
[\beta \otimes 1]^{0,2} \in H_{d R}^{2}(\widehat{T} / \widehat{\Delta}) / F^{1} H_{d R}^{2}(\widehat{T} / \widehat{\Delta}),
$$

where $\widehat{\Delta}$ is the completion of $\Delta$ at 0 , and $\widehat{\Delta}$ is the completion of $T$ along $S$.

But the scheme theoretic Noether-Lefschetz locus is defined precisely by the vanishing of $[\beta \otimes 1]^{0,2}$, and we assumed this is $\{0\} \subset \Delta$. Therefore $\left.f\right|_{\Delta}$ is the constant map to $0 \in \Delta$.

By Proposition A.3, $\mathcal{M}^{\star}$ is a moduli space of stable sheaves on $S$. By [38, Corollary 3.5], $\mathcal{M}^{\star}$ is nonsingular, nonempty, and deformation equivalent to $\operatorname{Hilb}^{g}(S)$, where $2 g-2=\beta^{2}$. In particular, $\mathcal{M}^{\star}$ has canonical orientation data.

We expect the Chow support map to the complete linear system in class $\beta$,

$$
\mathcal{M}^{\star} \underset{43}{\longrightarrow}|\mathcal{O}(\beta)|
$$


to factor through a Lagrangian fibration onto a projective space

$$
\mathbb{P}^{g} \subset|\mathcal{O}(\beta)| .
$$

The proof in most cases is well-known:

- If $\beta^{2}>0$ and $\beta$ is nef, then (A.4) is the classical Beauville integrable system, and the image $\mathbb{P}^{g}$ is the whole linear system $|\mathcal{O}(\beta)|$. The generic element $C$ of $|\mathcal{O}(\beta)|$ is a nonsingular irreducible curve of genus

$$
g=1+\beta^{2} / 2,
$$

and the fibre of (A.4) over $C$ is the nonsingular Lagrangian torus $\mathrm{Pic}_{g} C \subset \mathcal{M}^{\star}$.

- If $\beta^{2}=0$ and $\beta$ is nef of divisibility $m>0$, then $\beta / m$ is the class of a fibre in an elliptic fibration $S \rightarrow \mathbb{P}^{1}$. The sheaves parameterized by $\mathcal{M}^{\star}$ are (the pushforward to $S$ of) rank $m$ sheaves on a single fibre, and the fibration (A.4) maps this sheaf to its support in $\mathbb{P}^{1}=|\mathcal{O}(\beta / m)|$. In turn this $\mathbb{P}^{1}$ embeds in $\mathbb{P}^{m}=|\mathcal{O}(\beta)|$ as in (A.5) by the $m$ th Veronese embedding.

- If $\beta^{2}=-2, \mathcal{M}^{\star}$ is a single point, and the claim is trivial.

- If $\beta^{2}<-2, \mathcal{M}^{\star}$ is empty.

The remaining case where $\beta^{2} \geq 0$ with $\beta$ not nef is likely to follow from the above cases after using wall-crossing and the methods of [1, Section 11]. Alternatively, it is not hard to show directly that the fibres of the map (A.4) are Lagrangian; that the image should then be $\mathbb{P}^{g}$ is a standard conjecture (proved by Hwang when the image is nonsingular). We leave the matter open here.

Finally, for the cases treated above, we can follow the calculation of [21, Section 8.3] (which in turn follows [14]) to determine the refined Gopakumar-Vafa invariants using the perverse Leray filtration for the 
fibration 26 At the level of Poincaré polynomials, the generating function 27 obtained is:

$\prod_{m \geq 1} \frac{1}{\left(1-t_{L} t_{R} q^{m}\right)\left(1-t_{L}^{-1} t_{R} q^{m}\right)\left(1-t_{L} t_{R}^{-1} q^{m}\right)\left(1-t_{L}^{-1} t_{R}^{-1} q^{m}\right)\left(1-q^{m}\right)^{20}}$.

Substituting $(u, v)$ for $\left(t_{L}, t_{R}\right)$ gives the refined KKV generating function (5.1) of KKP.

Acknowledgements. Grateful thanks are due to A. Bayer for generous assistance in explaining the results of [1] and helping with (A.4).

\section{REFERENCES}

[1] A. Bayer and E. Macrì, MMP for moduli of sheaves on K3s via wall-crossing: nef and movable cones, Lagrangian fibrations, arXiv:1301.6968.

[2] A. Beauville, Counting rational curves on K3 surfaces, Duke Math. J. 97 (1999), 99-108.

[3] S. Bloch, Semi-regularity and de Rham cohomology, Invent. Math. 17 (1972), $51-66$.

[4] V. Bussi, D. Joyce, and S. Meinhardt, On motivic vanishing cycles of critical loci, arXiv:1305.6428.

[5] M.C.N. Cheng, K3 Surfaces, N=4 Dyons, and the Mathieu Group M24, Comm. Num. Theor. Phys. 4 (2010), 623.

[6] M.C.N. Cheng, J.F.R. Duncan, S.M. Harrison, and S. Kachru, Equivariant K3 invariants, arXiv:1508.02047.

[7] J. Choi, S. Katz, and A. Klemm, The refined BPS index from stable pair invariants, Comm. Math. Phys. 328 (2014), 903-954.

[8] J. Denef and F. Loeser, Motivic Igusa zeta functions, J. Alg. Geom. 7 (1998), $505-537$.

[9] J. Denef and F. Loeser, Motivic exponential integrals and a motivic ThomSebastiani theorem, Duke Math. J. 99 (1999), 285-309.

[10] I. Dolgachev and S. Kondo, Moduli of K3 surfaces and complex ball quotients, Lectures in Istambul, math.AG/0511051.

[11] T. Eguchi, H. Ooguri and Y. Tachikawa, Notes on the K3 Surface and the Mathieu group $M_{24}$, Exper. Math. 20 (2011), 91).

[12] M. Gaberdiel, S. Hohenegger, R. Volpato, Symmetries of K3 sigma models, Comm. Num. Theor. Phys. 6 (2012), 1-50.

[13] L. Göttsche, The Betti numbers of the Hilbert schemes of points on a smooth projective surface, Math. Ann. 286 (1990), 193-207.

[14] S. Hosono, M.-H. Saito, and A. Takahashi, Relative Lefschetz action and BPS state counting, Int. Math. Res. Notices 15 (2001), 783-816.

\footnotetext{
${ }^{26}$ The cited references use the fibration $\operatorname{Hilb}^{g}(S) \rightarrow \mathbb{P}^{g}$ induced by an elliptic fibration $S \rightarrow \mathbb{P}^{1}$. Our fibration $\mathcal{M}^{\star} \rightarrow \mathbb{P}^{g}$ can be deformed to this without changing the results.

${ }^{27}$ See the equation before [21, Equation (8.3)], before the specialization $t_{R}=-1$.
} 
[15] M.-X. Huang, A. Klemm and M. Poretschkin, Refined stable pair invariants for E-, $M$ - and $[p, q]$-strings, JHEP 1311 (2013), 112.

[16] D. Joyce, A classical model for derived critical loci, arXiv:1304.4508.

[17] S. Katz, A. Klemm, C Vafa, M-theory, topological strings, and spinning black holes, Adv. Theor. Math. Phys. 3 (1999), 1445-1537.

[18] S. Katz, Genus zero Gopakumar-Vafa invariants of contractible curves, J. Diff. Geom. 79 (2008), 185-195.

[19] S. Katz, Refined and motivic BPS invariants of stable pairs, String Math 2014 (Edmonton, Canada).

[20] T. Kawai and K Yoshioka, String partition functions and infinite products, Adv. Theor. Math. Phys. 4 (2000), 397-485.

[21] Y. Kiem and J. Li, Categorification of Donaldson-Thomas invariants via perverse sheaves, arXiv:1212.6444.

[22] A. Klemm, D. Maulik, R. Pandharipande, and E. Scheidegger, NoetherLefschetz theory and the Yau-Zaslow conjecture, J. AMS 23 (2010), 1013-1040.

[23] M. Kontsevich and Y. Soibelman, Stability structures, motivic DonaldsonThomas invariants and cluster transformations, arXiv:0811.2435.

[24] E. Looijenga, Motivic measures, Astérisque 276 (2002), 267-297.

[25] D. Maulik and R. Pandharipande, Gromov-Witten theory and NoetherLefschetz theory, in A celebration of algebraic geometry, Clay Mathematics Proceedings 18, 469-507, AMS (2010).

[26] R. Pandharipande and A. Pixton, Gromov-Witten/Pairs correspondence for the quintic 3-fold, arXiv:1206.5490.

[27] R. Pandharipande and R. P. Thomas, Curve counting via stable pairs in the derived category, Invent. Math. 178 (2009), 407-447.

[28] R. Pandharipande and R. P. Thomas, Stable pairs and BPS invariants, J. AMS 23 (2010), 267-297.

[29] R. Pandharipande and R. P. Thomas, 13/2 ways of counting curves, arXiv:1111.1552.

[30] R. Pandharipande and R. P. Thomas, The Katz-Klemm-Vafa conjecture for K3 surfaces, arXiv:1404.6698.

[31] T. Shioda and T. Katsura, On Fermat varieties, Tohoku Math. J. 31 (1979), 97-115.

[32] B. Szendröi, Nekrasov's partition function and refined Donaldson-Thomas theory: the rank one case, SIGMA 8 (2012), 1-16.

[33] B. Szendröi, Cohomological Donaldson-Thomas theory, arXiv:1503.07349.

[34] Y. Toda, Stable pairs on local K3 surfaces, J. Diff. Geom. 92 (2012), 285-371.

[35] C. Vafa and E. Witten, Dual string pairs with $N=1$ and $N=2$ supersymmetry in four-dimensions, Nucl. Phys. Proc. Suppl. 46 (1996), 225.

[36] C. T. C. Wall, On the orthogonal groups of unimodular quadratic forms, Math. Ann. 147 (1962), 328-338.

[37] S.-T. Yau and E. Zaslow, BPS states, string duality, and nodal curves on K3, Nucl. Phys. B457 (1995), 484-512.

[38] K. Yoshioka, Stability and the Fourier-Mukai transform II, Comp. Math. 145 (2009), 112-142. 
Department of Mathematics

University of Illinois

katz@math.uiuc.edu

Physikalisches Institut

Universität Bonn

aklemm@physik.uni-bonn.de

Departement Mathematik

ETH Zürich

rahul@math.ethz.ch 